\title{
GENERALIZED NAVIER-STOKES EQUATIONS WITH NONLINEAR ANISOTROPIC VISCOSITY
}

\author{
H. B. DE OLIVEIRA ${ }^{(1,2)}$ \\ (1) FCT - Universidade do Algarve, Faro, Portugal \\ ${ }^{(2)}$ CMAFCIO - Universidade de Lisboa, Portugal
}

\begin{abstract}
The purpose of this work is to study the generalized Navier-Stokes equations with nonlinear viscosity that, in addition, can be fully anisotropic. Existence of very weak solutions is proved for the associated initial and boundary-value problem, supplemented with no-slip boundary conditions. We show that our existence result is optimal in some directions provided there is some compensation in the remaining directions. A particular simplification of the problem studied here, reduces to the Navier-Stokes equations with (linear) anisotropic viscosity used to model either the turbulence or the Ekman layer in atmospheric and oceanic fluid flows.
\end{abstract}

Key words and phrases: Nonlinear anisotropic viscosity, Generalized Navier-Stokes, existence

2010 Mathematics Subject Classification: 35Q35, 76D05, 35Q30, 76D03, 35D99

\section{INTRODUCTION}

In the literature of large-scale flows, Navier-Stokes equations with anisotropic viscosity are used to model oceanographic and atmospheric fluid flows for which the horizontal extents are much larger than the vertical ones. For instance, a simple model used to describe turbulence in large-scale flows considers the Reynoldsaveraged Navier-Stokes equations,

$$
\begin{aligned}
& \operatorname{div} \mathbf{u}=0, \\
& \frac{\partial \mathbf{u}}{\partial t}+\operatorname{div}(\mathbf{u} \otimes \mathbf{u})=\mathbf{f}-\frac{1}{\rho} \boldsymbol{\nabla} p+\nu \operatorname{div}(\mathbf{D}(\mathbf{u}))+\operatorname{div}(\mathbf{R}),
\end{aligned}
$$

with the Reynolds stresses $\mathbf{R}$ modelled as follows,

$$
\mathbf{R}=\left[\begin{array}{ccc}
2 A_{H} \frac{\partial u_{1}}{\partial x_{1}} & A_{H}\left(\frac{\partial u_{2}}{\partial x_{1}}+\frac{\partial u_{1}}{\partial x_{2}}\right) & A_{H} \frac{\partial u_{3}}{\partial x_{1}}+A_{V} \frac{\partial u_{1}}{\partial x_{3}} \\
A_{H}\left(\frac{\partial u_{1}}{\partial x_{2}}+\frac{\partial u_{2}}{\partial x_{1}}\right) & 2 A_{H} \frac{\partial u_{2}}{\partial x_{2}} & A_{H} \frac{\partial u_{3}}{\partial x_{2}}+A_{V} \frac{\partial u_{2}}{\partial x_{3}} \\
A_{V} \frac{\partial u_{1}}{\partial x_{3}}+A_{H} \frac{\partial u_{3}}{\partial x_{1}} & A_{V} \frac{\partial u_{2}}{\partial x_{3}}+A_{H} \frac{\partial u_{3}}{\partial x_{2}} & 2 A_{H} \frac{\partial u_{3}}{\partial x_{3}}
\end{array}\right] .
$$

It should be noted that all non constant quantities in the equations (1.1)-(1.3), as the velocity vector field $\mathbf{u}=\left(u_{1}, u_{2}, u_{3}\right)$, the pressure $p$ and the external forces field $\mathbf{f}=\left(f_{1}, f_{2}, f_{3}\right)$ are, in fact, averages that result by the application of the Reynolds average decomposition. For instance, the velocity field, say $\mathbf{v}$, is decomposed into an average velocity $\mathbf{u}$ and a fluctuating velocity $\mathbf{u}^{\prime}: \mathbf{v}=\mathbf{u}+\mathbf{u}^{\prime}$, with $\mathbf{u}:=\overline{\mathbf{v}}$. The averaged tensor $\mathbf{D}(\mathbf{u})$ is the symmetric part of the averaged gradient $\boldsymbol{\nabla} \mathbf{u}$, and the positive constant $\nu$ is the kinematic viscosity and expresses the ratio of the internal forces in the fluid (dynamic viscosity) to the mass density

E-mail address: holivei@ualg.pt.

Date: June 4, 2019.

The author was supported by the Grant no. SFRH/BSAB/135242/2017 of the Portuguese Foundation for Science and Technology, Portugal. He was also partially supported by the Portuguese Foundation for Science and Technology, Portugal, under the project: UID/MAT/04561/2019. 
$\rho$, assumed here to be constant and positive. The extra term in (1.2) results as an output of the Reynolds average decomposition of the original Navier-Stokes equations and accounts for the average changes in $\mathbf{u}^{\prime}$ due to the particle transport with the fluid movement: $\mathbf{R}:=-\overline{\mathbf{u}^{\prime} \otimes \mathbf{u}^{\prime}}$. The coefficients $A_{H}$ and $A_{V}$ given in (1.3) denote the horizontal and vertical turbulent viscosities, which are supposed to be both nonnegative. Estimates of the turbulent viscosity in the atmosphere and in the ocean show that the values of $A_{H}$ and $A_{V}$ greatly exceed the kinematics viscosity $\nu$. Therefore the viscosity terms can be neglected from (1.2) so that the mean flow can be fairly described by the following equation,

$$
\frac{\partial \mathbf{u}}{\partial t}+\operatorname{div}(\mathbf{u} \otimes \mathbf{u})=\mathbf{f}-\frac{1}{\rho} \nabla p+\left(A_{H} \triangle_{H} u_{1}, A_{H} \triangle_{H} u_{2}, A_{V} \partial_{3}^{2} u_{3}\right),
$$

where $\triangle_{H}:=\frac{\partial^{2}}{\partial x_{1}^{2}}+\frac{\partial^{2}}{\partial x_{2}^{2}}$ and $\partial_{3}^{2}:=\frac{\partial^{2}}{\partial x_{3}^{2}}$. Note that if $A_{H}=A_{V}=A$, then the mean flow equation (1.2) is identical to that of the classical Navier-Stokes equations with an effective viscosity $\nu+A$, or with an effective viscosity of just $A$ if we neglect the viscosity terms as in (1.4). Similar equations to (1.4) also appear in the study of the friction region, called the Ekman layer, in geophysical fluid dynamics. Such friction layers, in conjunction with the constraints of the Taylor-Proudman theorem are shown to exert a profound influence on the dynamics of the flow far from the regions which are directly affected by viscosity. See, for instance, [20, Chapter 4] for the application of (1.4) in the governing equations of atmospheric and oceanic flows.

Motivated by these applications, we will consider in this work the following equation that encompasses many other situations of anisotropic diffusion,

$$
\frac{\partial \mathbf{u}}{\partial t}+\operatorname{div}(\mathbf{u} \otimes \mathbf{u})=\mathbf{f}-\nabla p+\sum_{i=1}^{N} \nu_{i} D_{i}\left(\left|D_{i} \mathbf{u}\right|^{q_{i}-2} D_{i} \mathbf{u}\right) .
$$

The directional derivative $D_{i} \mathbf{u}$ is defined by $D_{i} \mathbf{u}=\left(\partial_{i} u_{1}, \ldots, \partial_{i} u_{N}\right)$, where $\partial_{i} u_{j}=\frac{\partial u_{j}}{\partial x_{i}}$, and $N \geq 2$ is a general space dimension that we are now considering. The exponents $q_{i}$ and the coefficients $\nu_{i}$ are assumed to be constant with possible distinct values and such that $1<q_{i}<\infty$ and $\nu_{i}>0$ for all $i \in\{1, \ldots, N\}$. If we let $N=3, q_{1}=q_{2}=q_{3}=2, \nu_{1}=\nu_{2}=\nu_{3}=\nu$, then we obtain from (1.5) the classical Navier-Stokes equations for a renormalized pressure $P=\frac{p}{\rho}$. On the other hand, if besides $N=3$ and $q_{1}=q_{2}=q_{3}=2$, we consider $\nu_{1}=\nu_{2}=A_{H}$ and $\nu_{3}=A_{V}$, and we neglect the second-order cross derivatives between the horizontal and vertical components, i.e. if we assume $\frac{\partial^{2} u_{3}}{\partial x_{i}^{2}}=\frac{\partial^{2} u_{i}}{\partial x_{3}^{2}}=0$ for all $i \in\{1,2\}$, we can recover from (1.5) the momentum equation (1.4), again for a renormalized pressure. In fact, neglecting these second-order cross derivatives can be justified by a dimensional scale analysis in the governing equations of large-scale flows (see e.g. [6, Chapter 4]).

We consider the system formed by the equations (1.1) and (1.5) in a cylinder $Q_{T}:=\Omega \times[0, T]$, where $\Omega \subset \mathbb{R}^{N}$ is a bounded domain with its boundary denoted by $\partial \Omega$, and $T>0$ is some fixed time. The boundary of $Q_{T}$ shall be denoted by $\Gamma_{T}:=\partial \Omega \times[0, T]$. System of equations formed by (1.1) and (1.5) is supplemented by the following initial and boundary conditions,

$$
\begin{aligned}
& \mathbf{u}=\mathbf{u}_{0} \quad \text { in } \quad \Omega \quad \text { for } \quad t=0 \\
& \mathbf{u}=\mathbf{0} \quad \text { on } \quad \Gamma_{T} .
\end{aligned}
$$

A generic element of $\Omega$ shall be denoted by $\mathbf{x}=\left(x_{1}, \ldots, x_{N}\right)$, while the elements of $Q_{T}$ are denoted by $(\mathbf{x}, t)$. The problem we study in this work is the following: given two vector fields $\mathbf{f}=\mathbf{f}(\mathbf{x}, t)$ and $\mathbf{u}_{0}=\mathbf{u}_{0}(\mathbf{x})$, to find a vector field $\mathbf{u}=\mathbf{u}(\mathbf{x}, t)$ and a scalar field $p=p(\mathbf{x}, t)$ satisfying the system of equations posed by (1.1), (1.5) and (1.6)-(1.7)

To our best knowledge, the anisotropic problem formed by (1.1), (1.5) and (1.6)-(1.7) have been studied by the first time by Antontsev and Oliveira $[2,3]$. In [2] it was studied the existence of weak and very weak solutions for the stationary problem, whereas in [3] we have studied the evolutionary problem with respect to the existence of weak solutions and their asymptotic behavior. The present work is devoted to study the existence of very weak solutions to the problem posed by the equations (1.1), (1.5) and (1.6)-(1.7). We distinguish the notion of very weak solutions from the notion of weak solutions in the following sense. In the definition of weak solutions, solutions and test functions belong to the same function space, whereas, in the definition of very weak solutions, test functions belong to a much smoother function space then solutions. 
A distinct type of anisotropic Navier-Stokes equations was studied by Soltanov in [24]. Motivated by a problem suggested earlier in [15, Problem 2.11.24], the equations considered in [24] have the anisotropic diffusion term written in the form $\sum_{i=1}^{N} D_{i}\left(|\mathbf{u}|^{q_{i}-2} D_{i} \mathbf{u}\right)$ instead. There are also some other works that use the functional framework of anisotropic function spaces, but the considered problem is based on the (isotropic) classical Navier-Stokes equations (see e.g. [11]). However, in three dimensions, the particular case of considering (1.4) in the governing equations has been studied firstly by Chemin et al. [4] and Iftimie [12], and then by many other authors (see e.g. $[5,18,19,29,30]$ ).

The isotropic version of the problem formed by (1.1), (1.5) and (1.6)-(1.7), i.e. the case when in equation (1.5) $\nu_{i}=\nu$ and $q_{i}=q$ for all $i \in\{1, \ldots, N\}$, is being studied in the context of Rheology, at least since the experimental works by Ostwald [17] and de Waele [27]. In this case, the momentum equation is

$$
\frac{\partial \mathbf{u}}{\partial t}+(\mathbf{u} \cdot \boldsymbol{\nabla}) \mathbf{u}=\mathbf{f}-\frac{1}{\rho} \boldsymbol{\nabla} p+\nu \operatorname{div}\left(|\mathbf{D}(\mathbf{u})|^{q-2} \mathbf{D}(\mathbf{u})\right),
$$

where, here, $q$ is the power-law index that characterizes the flow. Existence and uniqueness results to the correspondingly problem for the momentum equation (1.8), were established by many authors, among them the breakthroughs achieved by Ladyzhenskaya [13, 14], Lions [15], Wölf [28] and Diening et al. [7] (see also Málek et al. [16] and Zhikov [31]).

Navier-Stokes equations with other anisotropic terms have been studied in the literature. For instance, Navier-Stokes equations with small initial data in suitable anisotropic function spaces were considered in [11], and with anisotropic feedback forces fields have been studied in [1]. There are also some works in which some anisotropic regularity criteria for the Navier-Stokes equations, extending the well-known Serrin condition, were considered (see e.g. [21]).

The plan of this paper is the followings. Section 2 is dedicated to introduce the main concepts of the anisotropic function spaces we are going to work with, as well to define the notion of very weak solution to our problem. In Section 3 is presented the main result of this work in Theorem 3.1, but first we make there a brief historical review of past results related to our. The rest of the article, i.e. from Section 4 onwards, is devoted to the proof of Theorem 3.1.

The notation used throughout this article and the main notions of the considered (isotropic) function spaces are largely standard in the literature of Partial Differential Equations and in Mathematical Fluid Mechanics as well. We address the reader to the monographs $[9,14,15,16]$ for any question related to that matter.

\section{Anisotropic FUnCtion SPACES}

Due to the presence of possibly different exponents $q_{i}$ for distinct directions, we need to consider the solutions to the problem formed by (1.1), (1.5) and (1.6)-(1.7) in some anisotropic Sobolev space. We define the vector $\mathbf{q}$ in $\mathbb{R}^{N}$, whose components are the exponents of the anisotropic diffusion term considered in (1.5), by

$$
\mathbf{q}:=\left(q_{1}, \cdots, q_{N}\right), \quad 1<q_{i}<\infty \quad \forall i \in\{1, \ldots, N\}
$$

and let us set

$$
\beta:=\max _{i \in\{1, \ldots, N\}} q_{i} \text { and } \alpha:=\min _{i \in\{1, \ldots, N\}} q_{i} .
$$

To avoid any confusion that $\mathbf{q}$ is in fact multi-component, in the rest of our work we will emphasize this meaning by writing an arrow over $\mathbf{q}$ : $\overrightarrow{\mathbf{q}}$. For simplicity, we assume throughout the text that the components of $\overrightarrow{\mathbf{q}}=\left(q_{1}, q_{2}, \ldots, q_{N}\right)$ satisfy to

$$
\alpha=q_{1} \leq q_{2} \leq \cdots \leq q_{N}=\beta .
$$

Considering the unidirectional Sobolev spaces

$$
\mathbf{W}_{i}^{1, q_{i}}(\Omega)=\left\{\mathbf{v} \in \mathbf{W}^{1,1}(\Omega): \quad D_{i} \mathbf{v} \in \mathbf{L}^{q_{i}}(\Omega)\right\}, \quad i \in\{1, \ldots, N\},
$$

which are Banach spaces for the norm

$$
\|\mathbf{v}\|_{\mathbf{W}_{i}^{1, q_{i}}(\Omega)}=\|\mathbf{v}\|_{\mathbf{L}^{1}(\Omega)}+\left\|D_{i} \mathbf{v}\right\|_{\mathbf{L}^{q_{i}}(\Omega)}, \quad i \in\{1, \ldots, N\},
$$


we define the anisotropic Sobolev space $\mathbf{W}^{1,} \overrightarrow{\mathbf{q}}(\Omega)$ as follows,

$$
\mathbf{W}^{1, \overrightarrow{\mathbf{q}}}(\Omega):=\bigcap_{i=1}^{N} \mathbf{W}_{i}^{1, q_{i}}(\Omega),
$$

with the norm defined by

$$
\|\mathbf{v}\|_{\mathbf{W}^{1, \overrightarrow{\mathbf{q}}}(\Omega)}=\|\mathbf{v}\|_{\mathbf{L}^{1}(\Omega)}+\sum_{i=1}^{N}\left\|D_{i} \mathbf{v}\right\|_{\mathbf{L}^{q_{i}}(\Omega)} .
$$

An important limitation of the anisotropic Sobolev space $\mathbf{W}^{1,} \mathbf{\mathbf { q }}(\Omega)$, is that, for bounded domains $\Omega$, the validity of Sobolev imbeddings is restricted to rectangular domains (see e.g. [10]). In fact, for rectangular domains $\Omega$, the following imbedding is continuous (cf. [23, Theorem 1])

$$
\mathbf{W}^{1, \overrightarrow{\mathbf{q}}}(\Omega) \hookrightarrow \mathbf{L}^{s}(\Omega) \quad \text { for any } s:\left\{\begin{array}{l}
1 \leq s \leq \bar{q}^{*}, \quad \sum_{j=1}^{N} \frac{1}{q_{j}}>1 \\
1 \leq s<\infty, \quad \sum_{j=1}^{N} \frac{1}{q_{j}} \leq 1,
\end{array}\right.
$$

where $\bar{q}^{*}$ denotes the Sobolev conjugate of $\bar{q}$, the harmonic mean of $q_{1}, \ldots, q_{N}$ :

$$
\bar{q}^{*}:=\frac{N \bar{q}}{N-\bar{q}}, \quad \bar{q}:=\frac{N}{\sum_{j=1}^{N} \frac{1}{q_{j}}}, \quad \sum_{j=1}^{N} \frac{1}{q_{j}}>1 \quad \Rightarrow \quad \bar{q}^{*}=\frac{N}{\sum_{j=1}^{N} \frac{1}{q_{j}}-1} .
$$

Moreover, the imbedding (2.1) is compact ( $c f$. [23, Theorem 2]), and we denote this fact by writing

$$
\mathbf{W}^{1, \overrightarrow{\mathbf{q}}}(\Omega) \hookrightarrow \hookrightarrow \mathbf{L}^{s}(\Omega) \text { for any } s: 1 \leq s<\bar{q}^{*} .
$$

In some situations it is possible to remove the restrictions on the shape's domain and to enlarge the interval of $s$ for the validity of $(2.1)$ and (2.2). Let us see this fact by defining

$$
\mathbf{W}_{0}^{1, \overrightarrow{\mathbf{q}}}(\Omega):=\text { closure of } \mathbf{C}_{0}^{\infty}(\Omega) \text { in the anisotropic norm of } \mathbf{W}^{1, \overrightarrow{\mathbf{q}}}(\Omega) \text {. }
$$

In this case, we have $\left(c f\right.$. [8, Theorem 1]), in the interesting case of $\sum_{j=1}^{N} \frac{1}{q_{j}}>1$, that

$$
\begin{aligned}
& \mathbf{W}_{0}^{1, \overrightarrow{\mathbf{q}}}(\Omega) \hookrightarrow \mathbf{L}^{s}(\Omega) \quad \text { for any } s: 1 \leq s \leq q_{a}^{*}, \\
& \mathbf{W}_{0}^{1, \overrightarrow{\mathbf{q}}}(\Omega) \hookrightarrow \hookrightarrow \mathbf{L}^{s}(\Omega) \text { for any } s: 1 \leq s<q_{a}^{*},
\end{aligned}
$$

where $q_{a}^{*}$ is the critical exponent defined by

$$
q_{a}^{*}:=\max \left\{\bar{q}^{*}, \beta\right\} .
$$

Remark 2.1. Note that, for $N=2, \bar{q}^{*}>\beta$ and therefore $q_{a}^{*}=\bar{q}^{*}$. But, if $N>2$, it may well happen that $\beta>\bar{q}^{*}$. In fact, for $N>2$,

$$
\beta>\bar{q}^{*} \Leftrightarrow \bar{q}<\frac{\beta N}{\beta+N} \Leftrightarrow \sum_{j=1, q_{j} \neq \beta}^{N} \frac{1}{q_{j}}>1+\frac{N-1}{\beta} .
$$

This means that, in typical situations when $N>2, q_{a}^{*}=\beta$ if, at least, one of the components $q_{i}$ of $\overrightarrow{\mathbf{q}}$ is too far apart from the others. For instance, when $N=3, \overrightarrow{\mathbf{q}}=\left(\frac{10}{7}, \frac{20}{7}, \beta\right)$, we have $\beta>\bar{q}^{*}$ if and only if $\beta>40$. In this example, the first two components $q_{1}$ and $q_{2}$ are relatively close to each other, but we may have all the three components $q_{1}, q_{2}$ and $q_{3}$ sufficiently far apart one from each other, as shows the example $\overrightarrow{\mathbf{q}}=\left(\frac{1001}{1000}, \frac{1001}{2}, \beta\right)$ for which $\beta>\bar{q}^{*}$ if and only $\beta>2002$.

As a particular case of (2.3), it can be derived the following result, essentially due to Troisi [26] and extended in $[8,22]$.

Lemma 2.1. Let $\Omega \subset \mathbb{R}^{N}$ be an open bounded domain with a Lipschitz-continuous boundary $\partial \Omega$. Then for any $\mathbf{u} \in \mathbf{W}_{0}^{1, \overrightarrow{\mathbf{q}}}(\Omega)$

$$
\|\mathbf{u}\|_{\mathbf{L}^{s}(\Omega)} \leq C\left(\prod_{i=1}^{N}\left\|D_{i} \mathbf{u}\right\|_{\mathbf{L}^{q_{i}}(\Omega)}\right)^{\frac{1}{N}}
$$

for $s \in\left[1, q_{a}^{*}\right]$ if $\sum_{i=1}^{N} \frac{1}{q_{i}}>1$, or $s \in[1, \infty)$ otherwise, and where $C=C\left(\Omega, N, s, q_{i}\right)$ is a positive constant. 
Proof. The proof combines Theorem 1.2 of [26], Theorem 3.1 of [22] and Theorem 1 of [8].

Let us now recall the classical function spaces of Mathematical Fluid Mechanics,

$$
\begin{aligned}
& \mathcal{V}:=\left\{\mathbf{v} \in \mathbf{C}_{0}^{\infty}(\Omega): \operatorname{div} \mathbf{v}=0\right\} \\
& \mathbf{H}:=\text { closure of } \mathcal{V} \text { in the norm of } \mathbf{L}^{2}(\Omega), \\
& \mathbf{V}_{q}:=\text { closure of } \mathcal{V} \text { in } \mathbf{W}^{1, q}(\Omega)
\end{aligned}
$$

We define the anisotropic analogue of $\mathbf{V}_{q}$ as follows,

$$
\mathbf{V}_{\overrightarrow{\mathrm{q}}}:=\bigcap_{i=1}^{N} \mathbf{V}_{q_{i}}
$$

where $\mathbf{V}_{q_{i}}:=$ closure of $\mathcal{V}$ in the norm of $\mathbf{W}_{i}^{1, q_{i}}(\Omega)$. Now we consider the unidirectional Bochner space,

$$
\mathrm{L}^{q_{i}}\left(0, T ; \mathbf{V}_{q_{i}}\right):=\left\{\mathbf{v}:[0, T] \rightarrow \mathbf{V}_{q_{i}} \mid \mathbf{v} \in \mathbf{L}^{1}\left(Q_{T}\right), D_{i} \mathbf{v} \in \mathbf{L}^{q_{i}}\left(Q_{T}\right) \forall i \in\{1, \ldots, N\}\right\},
$$

which is a Banach space for the norm

$$
\|\mathbf{v}\|_{L^{q_{i}}\left(0, T ; \mathbf{V}_{q_{i}}\right)}:=\|\mathbf{v}\|_{\mathbf{L}^{1}\left(Q_{T}\right)}+\left\|D_{i} \mathbf{v}\right\|_{\mathbf{L}^{q_{i}}\left(Q_{T}\right)} .
$$

Then we define the anisotropic Banach space

$$
\mathrm{L}^{\overrightarrow{\mathrm{q}}}\left(0, T ; \mathbf{V}_{\overrightarrow{\mathrm{q}}}\right):=\bigcap_{i=1}^{N} \mathrm{~L}^{q_{i}}\left(0, T ; \mathbf{V}_{q_{i}}\right),
$$

whose norm is defined by

$$
\|\mathbf{v}\|_{L^{\overrightarrow{\mathbf{q}}\left(0, T ; \mathbf{V}_{\overrightarrow{\mathrm{q}}}\right)}}:=\|\mathbf{v}\|_{\mathbf{L}^{1}\left(Q_{T}\right)}+\sum_{i=1}^{N}\left\|D_{i} \mathbf{v}\right\|_{\mathbf{L}^{q_{i}}\left(Q_{T}\right)} .
$$

Note that, for a bounded domain $\Omega$ and for a finite $T$, the following continuous imbeddings hold

$$
\mathrm{L}^{\beta}\left(0, T ; \mathbf{V}_{\beta}\right) \hookrightarrow \mathrm{L}^{\overrightarrow{\mathrm{q}}}\left(0, T ; \mathbf{V}_{\overrightarrow{\mathrm{q}}}\right) \hookrightarrow \mathrm{L}^{\alpha}\left(0, T ; \mathbf{V}_{\alpha}\right) .
$$

Therefore, as a closed subspace of $\mathrm{L}^{\alpha}\left(0, T ; \mathbf{V}_{\alpha}\right)$, the anisotropic parabolic space $\mathrm{L}^{\overrightarrow{\mathrm{q}}}\left(0, T ; \mathbf{V}_{\overrightarrow{\mathrm{q}}}\right)$ is separable and reflexive. By $\mathrm{L}^{q_{i}^{\prime}}\left(0, T ; \mathbf{V}_{q_{i}}^{\prime}\right)$ and $\mathrm{L}^{\overrightarrow{\mathrm{q}}^{\prime}}\left(0, T ; \mathbf{V}_{\overrightarrow{\mathrm{q}}}^{\prime}\right)$, we denote the dual spaces of $\mathrm{L}^{q_{i}}\left(0, T ; \mathbf{V}_{q_{i}}\right)$ and $\mathrm{L}^{\overrightarrow{\mathrm{q}}}\left(0, T ; \mathbf{V}_{\overrightarrow{\mathrm{q}}}\right)$, respectively, and where $\mathbf{V}_{q_{i}}^{\prime}$ and $\mathbf{V}_{\overrightarrow{\mathrm{q}}}^{\prime}$ stay for the dual spaces of $\mathbf{V}_{q_{i}}$ and $\mathbf{V}_{\overrightarrow{\mathrm{q}}}$.

\section{THE MAIN RESUlT}

Regarding the issue of existence, which is our main goal in this work, we take some time to make a historical review of known results for the isotropic problem corresponding to the momentum equation (1.8). In Ladyzhenskaya [13, Theorem 1.1] was established the existence of weak solutions for $q \geq \frac{12}{5}$, in the case of $N=3$ and under the assumptions that $\mathbf{u}_{0} \in \mathbf{H}$ and $\mathbf{f} \in \mathrm{L}^{2}\left(Q_{T}\right)$. Then, Lions [15, Section 2.5] has improved and extended, to a general dimension $N \geq 2$, the existence result of [13]. Under the assumptions that $\mathbf{u}_{0} \in \mathbf{H}$ and $\mathbf{f} \in \mathrm{L}^{q^{\prime}}\left(0, T ; \mathbf{V}_{q}^{\prime}\right), \quad[15$, Théorème 2.5.1] asserts the existence of weak solutions $\mathbf{u} \in \mathrm{L}^{q}\left(0, T ; \mathbf{V}_{q}\right) \cap \mathrm{L}^{\infty}(0, T ; \mathbf{H})$. Both proofs in [13] and [15] use Galerkin approximations and compactness arguments together with the theory of monotone operators. The improvement of [15, Théorème 2.5.1] relies in the fact that the continuous imbedding

$$
\mathrm{L}^{q}\left(0, T ; \mathbf{V}_{q}\right) \cap \mathrm{L}^{\infty}(0, T ; \mathbf{H}) \hookrightarrow \mathrm{L}^{q \frac{N+2}{N}}\left(Q_{T}\right)
$$

implies the boundedness of $\mathbf{u} \otimes \mathbf{u}: \mathbf{D}(\mathbf{v})$ in $\mathbf{L}^{1}\left(Q_{T}\right)$ for all $\mathbf{u}, \mathbf{v} \in \mathrm{L}^{q}\left(0, T ; \mathbf{V}_{q}\right) \cap \mathrm{L}^{\infty}(0, T ; \mathbf{H})$, provided that

$$
q \geq \frac{3 N+2}{N+2} .
$$

A demand in the search of results of existence for lower values of q was subsequently carried out by several authors. In particular, Nečas and his collaborators developed a systematic program with much more Fluid Mechanics insights to analyze the shear thinning case, i.e. the case for which $1<q<2$ (see e.g. [16]). 
However, it was only almost 40 years later, that Wolf [28] has proved the existence of very weak solution, i.e. solutions satisfying to

$$
\begin{aligned}
& -\int_{Q_{T}} \mathbf{u} \cdot \boldsymbol{\varphi}_{t} d \mathbf{x} d t+\int_{Q_{T}}(\mathbf{S}(\mathbf{D}(\mathbf{u}))-\mathbf{u} \otimes \mathbf{u}): \mathbf{D}(\boldsymbol{\varphi}) d \mathbf{x} d t \\
& =\int_{Q_{T}} \mathbf{f} \cdot \boldsymbol{\varphi} d \mathbf{x} d t+\int_{\Omega} \mathbf{u}_{0} \cdot \varphi(0) d \mathbf{x},
\end{aligned}
$$

but now for all $\boldsymbol{\varphi} \in \mathbf{C}^{\infty}\left(Q_{T}\right)$, with $\operatorname{div} \boldsymbol{\varphi}=0$ and $\operatorname{supp} \varphi \subset \subset \Omega \times[0, T)$. In (3.3), $\mathbf{S}$ is a generic monotonous tensor function of the symmetric part $\mathbf{D}(\mathbf{u})$ of the velocity gradient $\boldsymbol{\nabla} \mathbf{u}$, satisfying to suitable growth and coercivity conditions, and of which the (isotropic tensor) $|\mathbf{D}(\mathbf{u})|^{q-2} \mathbf{D}(\mathbf{u})$, with $1<q<\infty$, is a particular case. The author has used the assumptions that $\mathbf{u}_{0} \in \mathbf{H}, \mathbf{f}=-\operatorname{div} \mathbf{F}$ and $\mathbf{F} \in \mathbf{L}^{q^{\prime}}\left(Q_{T}\right)$ and observed the imbeddings (3.1) and $\mathrm{L}^{q}\left(0, T ; \mathbf{V}_{q}\right) \cap \mathrm{L}^{q \frac{N+2}{N}}\left(Q_{T}\right) \hookrightarrow \mathrm{L}^{s}\left(Q_{T}\right)$ imply that $\operatorname{div}(\mathbf{u} \otimes \mathbf{u}) \cdot \boldsymbol{\varphi}$ is bounded in $\mathbf{L}^{s}\left(Q_{T}\right)$ for all $\mathrm{L}^{\infty}$-truncation $\varphi$ of $\mathbf{u}$ and for some $s>1$, provided that

$$
q>2 \frac{N+1}{N+2} \text {. }
$$

In his proof, the author has used harmonic decomposition of $\mathrm{L}^{q}$-functions to decompose the pressure, into a measurable function and a singular part, to carry out the passage to the limit in the pressure terms. Finally in Diening et al. [7], the authors have improved the result [28] for smaller values of $q$,

$$
q>\frac{2 N}{N+2} .
$$

In that work the main tool was a well succeeded application of the Lipschitz-truncation method, but it was also very important the technique developed by Wolf to decompose the pressure.

Mathematically speaking, anisotropic models are good because allow one to improve known results for the isotropic models, at least in some directions of diffusion or for particular choices of all the diffusion coefficients. In particular, in [3, Theorem 3.1] we have proved the existence of weak solutions $\mathbf{u} \in$ $\mathrm{L}^{\infty}(0, T ; \mathbf{H}) \cap \mathrm{L}^{\overrightarrow{\mathrm{q}}}\left(0, T ; \mathbf{V}_{\overrightarrow{\mathbf{q}}}\right)$ to the problem formed by (1.1), (1.5) and (1.6)-(1.7), satisfying the following integral identity in the sense of distributions on $(0, T)$,

$$
\begin{aligned}
& \frac{d}{d t} \int_{\Omega} \mathbf{u}(t) \cdot \mathbf{v} d \mathbf{x}+\sum_{i=1}^{N} \int_{\Omega}\left|D_{i} \mathbf{u}(t)\right|^{q_{i}-2} D_{i} \mathbf{u}(t) \cdot D_{i} \mathbf{v} d \mathbf{x}+\int_{\Omega}[(\mathbf{u}(t) \cdot \nabla) \mathbf{u}(t)] \cdot \mathbf{v} d \mathbf{x}= \\
& \int_{\Omega} \mathbf{f}(t) \cdot \mathbf{v} d \mathbf{x},
\end{aligned}
$$

for any $\mathbf{v} \in \mathbf{V}_{\overrightarrow{\mathbf{q}}} \cap \mathbf{L}^{\theta}(\Omega)$ and where $\theta$ is such that $\frac{1}{q_{a}^{*}}+\frac{1}{\alpha}+\frac{1}{\theta}=1$. The main assumption to prove [3, Theorem 3.1], was a restriction on the lower bound of the critical anisotropic Sobolev exponent $q_{a}^{*}$,

$$
q_{a}^{*} \geq q_{*}:= \begin{cases}\frac{2 \alpha(\alpha-1)}{(\alpha+1)(\alpha-2)}, & 2<\alpha<3, \\ \frac{2 \alpha}{\alpha-1}, & \alpha \geq 3 .\end{cases}
$$

On the basis of the lower bound given by (3.5), are the anisotropic imbedding $\mathbf{V}_{\vec{q}} \hookrightarrow \mathbf{L}^{2 \alpha^{\prime}}(\Omega)$, which follows from (2.1), and the anisotropic counterpart of (3.1),

$$
\mathrm{L}^{\infty}(0, T ; \mathbf{H}) \cap \mathrm{L}^{\overrightarrow{\mathbf{q}}}\left(0, T ; \mathbf{V}_{\overrightarrow{\mathbf{q}}}\right) \hookrightarrow \mathbf{L}^{\rho}\left(Q_{T}\right) \quad \text { for } \quad \rho:=2+\alpha-\frac{2 \alpha}{q_{a}^{*}},
$$

which follows from (2.1) and parabolic interpolation. Observe that, by virtue of (3.5), [3, Theorem 3.1] improves the existence results $[13,15]$ in some directions of diffusion or for particular choices of all the diffusion coefficients (see the final part of [3, Section 3.4]). However, as observed in [3, Remark 3.2], if $q_{i}=q$ for all $i \in\{1, \ldots, N\}$, then condition (3.5) reduces to (3.2). On the other hand, the result [3, Theorem 3.1] cannot be compared with the results of $[7,28]$, because the notions of weak solutions these papers deal with are distinct. The goal of this work is to extend [3, Theorem 3.1] for lower bounds of $\alpha$ smaller than those provided by (3.5), introducing for that purpose the notion of very weak solution to the anisotropic problem posed by (1.1), (1.5) and (1.6)-(1.7). 
Definition 3.1. Let $N \geq 2$ and assume that $\mathbf{u}_{0} \in \mathbf{H}$ and $\mathbf{f} \in \mathbf{L}^{1}\left(Q_{T}\right)$. A vector field $\mathbf{u}$ is a very weak solution to the problem \{(1.1), (1.5), (1.6)-(1.7)\}, if:

(1) $\mathbf{u} \in \mathrm{L}^{\infty}(0, T ; \mathbf{H}) \cap \mathrm{L}^{\overrightarrow{\mathrm{q}}}\left(0, T ; \mathbf{V}_{\overrightarrow{\mathrm{q}}}\right)$;

(2) $\mathbf{u}(0)=\mathbf{u}_{0}$

(3) For every $\varphi \in \mathbf{C}^{\infty}\left(Q_{T}\right)$, with $\operatorname{div} \varphi=0$ and $\operatorname{supp} \varphi \subset \subset \Omega \times[0, T)$,

$$
\begin{aligned}
& -\int_{Q_{T}} \mathbf{u} \cdot \varphi_{t} d \mathbf{x} d t+\sum_{i=1}^{N} \int_{Q_{T}} \nu_{i}\left|D_{i} \mathbf{u}\right|^{q_{i}-2} D_{i} \mathbf{u} \cdot D_{i} \varphi d \mathbf{x} d t-\int_{Q_{T}} \mathbf{u} \otimes \mathbf{u}: \nabla \boldsymbol{\varphi} d \mathbf{x} d t \\
& =\int_{Q_{T}} \mathbf{f} \cdot \boldsymbol{\varphi} d \mathbf{x} d t+\int_{\Omega} \mathbf{u}_{0} \cdot \boldsymbol{\varphi}(0) d \mathbf{x} .
\end{aligned}
$$

We shall see in this work that, by considering the notion of very weak solutions stated at Definition 3.1, the lower bound of $q_{a}^{*}$ given at (3.5) can be considerably decreased. In fact, as we shall see in the proof of the our main result written bellow, we will establish the existence of very weak solutions in the case of

$$
q_{a}^{*}>\beta .
$$

Observe that, due to (2.4), the validity of (3.7) implies that $q_{a}^{*}=\bar{q}^{*}$. According to Remark 2.1, this case corresponds to a situation in which at least one of the components of $\overrightarrow{\mathbf{q}}$ is too far apart from the others.

Theorem 3.1. Let $\Omega$ be a bounded domain in $\mathbb{R}^{N}, N \geq 2$, and assume that

$$
\mathbf{f}=-\operatorname{div} \mathbf{F}, \quad \mathbf{F} \in \mathbf{L}^{\overrightarrow{\mathbf{q}}^{\prime}}\left(Q_{T}\right),
$$

$$
\mathbf{u}_{0} \in \mathbf{H}
$$

If the critical exponent $q_{a}^{*}$ satisfies to

$$
q_{a}^{*}>\max \{2, \beta\}
$$

then there exists a very weak solution $\mathbf{u} \in \mathrm{C}_{\mathrm{w}}([0, T] ; \mathbf{H}) \cap \mathrm{L}^{\overrightarrow{\mathrm{q}}}\left(0, T ; \mathbf{V}_{\overrightarrow{\mathrm{q}}}\right)$ to the problem formed by (1.1), (1.5) and (1.6)-(1.7) in the sense of Definition 3.1.

Here, by $\mathrm{C}_{\mathrm{w}}([0, T] ; \mathbf{H})$ we denote the subspace of $\mathrm{L}^{\infty}(0, T ; \mathbf{H})$ consisting of functions which are weakly continuous from $[0, T]$ into $\mathbf{H}$.

Remark 3.1. Note that if $q_{i}=q$ for all $i \in\{1, \ldots, N\}$, then, from condition (3.10), we recover the main restriction (3.4) on the existence of very weak solutions (see [7]) for the isotropic version of the problem formed by (1.1), (1.5) and (1.6)-(1.7).

In some parts of the proof of Theorem 3.1, we shall make use of the following relation to better identify each anisotropic diffusion component

$$
D_{i}\left(\left|D_{i} \mathbf{u}\right|^{q_{i}-2} D_{i} \mathbf{u}\right)=\operatorname{div}\left(\left|D_{i} \mathbf{u}\right|^{q_{i}-2} D_{i} \mathbf{u} \otimes \mathbf{e}_{i}\right),
$$

where $\left\{\mathbf{e}_{1}, \ldots, \mathbf{e}_{N}\right\}$ denotes the canonical basis of $\mathbb{R}^{N}$.

Proof. The proof of Theorem 3.1 will be carried out in the rest of this paper which will be split into several sections for better understanding. In the proof we use a regularization technique in the spirit of [31], we adapt the technique of decomposing the pressure developed in [28] and we use the Lipschitz approximation in the spirit of [7]. 
H.B. DE OLIVEIRA

\section{Existence of APproximate solutions}

Without loss of generality, we assume in the rest of this work that

$$
\nu_{i}=1 \quad \text { for all } i \in\{1, \ldots, N\} .
$$

For $\epsilon>0$, we consider the regularized problem

$$
\begin{aligned}
& \operatorname{div} \mathbf{u}_{\epsilon}=0 \quad \text { in } Q_{T} \\
& \frac{\partial \mathbf{u}_{\epsilon}}{\partial t}+\operatorname{div}\left(\mathbf{u}_{\epsilon} \otimes \mathbf{u}_{\epsilon}\right)=\mathbf{f}-\nabla p_{\epsilon}+\sum_{i=1}^{N} D_{i}\left(\left|D_{i} \mathbf{u}_{\epsilon}\right|^{q_{i}-2} D_{i} \mathbf{u}_{\epsilon}\right)+\epsilon \operatorname{div}\left(\left|\nabla \mathbf{u}_{\epsilon}\right|^{\gamma-2}\left|\nabla \mathbf{u}_{\epsilon}\right|\right) \quad \text { in } \quad Q_{T} \\
& \mathbf{u}_{\epsilon}=\mathbf{u}_{0} \quad \text { in } \Omega \text { for } t=0 \\
& \mathbf{u}_{\epsilon}=\mathbf{0} \quad \text { on } \Gamma_{T},
\end{aligned}
$$

where $\gamma$ is an exponent so large that, by one hand the convective term becomes a compact perturbation of the anisotropic Stokes problem, and on the other

$$
\int_{\Omega} \mathbf{u} \otimes \mathbf{u}: \nabla \mathbf{v} d \mathbf{x} \in \mathrm{L}^{1}(0, T) \quad \text { for } \mathbf{u} \in \mathrm{L}^{\infty}(0, T ; \mathbf{H}) \cap \mathrm{L}^{\overrightarrow{\mathbf{q}}}\left(0, T ; \mathbf{V}_{\overrightarrow{\mathbf{q}}}\right), \mathbf{v} \in \mathrm{L}^{\gamma}\left(0, T ; \mathbf{V}_{\gamma}\right)
$$

Moreover, $\gamma$ should be chosen in a way such that $\mathrm{L}^{\gamma}\left(0, T ; \mathbf{V}_{\gamma}\right) \hookrightarrow \mathrm{L}^{\overrightarrow{\mathbf{q}}}\left(0, T ; \mathbf{V}_{\overrightarrow{\mathbf{q}}}\right)$. In consequence of all this, we consider

$$
\gamma>\bar{\gamma}:=\max \left\{\frac{2 N}{N+2}, \beta, \frac{2 q_{a}^{*}}{\alpha\left(q_{a}^{*}-2\right)}+1\right\} .
$$

A vector function $\mathbf{u}_{\epsilon} \in \mathrm{L}^{\infty}(0, T ; \mathbf{H}) \cap \mathrm{L}^{\gamma}\left(0, T ; \mathbf{V}_{\gamma}\right)$ is a (very) weak solution to the problem (4.1)-(4.4), if

$$
\begin{aligned}
& -\int_{Q_{T}} \mathbf{u}_{\epsilon} \cdot \varphi_{t} d \mathbf{x} d t+\sum_{i=1}^{N} \int_{Q_{T}}\left|D_{i} \mathbf{u}_{\epsilon}\right|^{q_{i}-2} D_{i} \mathbf{u}_{\epsilon} \cdot D_{i} \boldsymbol{\varphi} d \mathbf{x} d t+\epsilon \int_{Q_{T}}\left|\nabla \mathbf{u}_{\epsilon}\right|^{\gamma-2} \nabla \mathbf{u}_{\epsilon}: \nabla \varphi d \mathbf{x} d t \\
& =\int_{Q_{T}} \mathbf{u}_{\epsilon} \otimes \mathbf{u}_{\epsilon}: \nabla \boldsymbol{\varphi} d \mathbf{x} d t+\int_{Q_{T}} \mathbf{f} \cdot \boldsymbol{\varphi} d \mathbf{x} d t+\int_{\Omega} \mathbf{u}_{0} \cdot \varphi(0) d \mathbf{x}
\end{aligned}
$$

for all $\varphi \in \mathbf{C}^{\infty}\left(Q_{T}\right)$, with $\operatorname{div} \varphi=0$ and $\operatorname{supp} \varphi \subset \subset \Omega \times[0, T)$.

Proposition 4.1. Let the assumptions of Theorem 3.1 be fulfilled and, in addition, assume that $\gamma$ obeys to (4.5). Then, for each $\epsilon>0$, there exists a very weak solution $\mathbf{u}_{\epsilon} \in \mathrm{L}^{\infty}(0, T ; \mathbf{H}) \cap \mathrm{L}^{\gamma}\left(0, T ; \mathbf{V}_{\gamma}\right)$ to the problem (4.1)-(4.4). In addition, every weak solution satisfies the following energy equality:

$$
\frac{1}{2}\left\|\mathbf{u}_{\epsilon}(t)\right\|_{\mathbf{H}}^{2}+\sum_{i=1}^{N} \int_{Q_{t}}\left|D_{i} \mathbf{u}_{\epsilon}\right|^{q_{i}} d \mathbf{x} d t+\epsilon \int_{Q_{t}}\left|\nabla \mathbf{u}_{\epsilon}\right|^{\gamma} d \mathbf{x} d t=\frac{1}{2}\left\|\mathbf{u}_{0}\right\|_{\mathbf{H}}^{2}+\int_{Q_{t}} \mathbf{F}: \nabla \mathbf{u}_{\epsilon} d \mathbf{x} d t
$$

for all $t \in(0, T)$.

Proof. The choice of $\gamma$ according to (4.5) allows one to use the theory of monotone operators (see e.g. [15, pp. 209-217]) to prove the existence. The energy relation (4.7) holds true, because, since the solutions and test functions are in the same function space, it is possible to test (4.2) by a solution $\mathbf{u}_{\epsilon}$.

Let $\mathbf{u}_{\epsilon} \in \mathrm{L}^{\infty}(0, T ; \mathbf{H}) \cap \mathrm{L}^{\gamma}\left(0, T ; \mathbf{V}_{\gamma}\right)$ be a weak solution to the problem (4.1)-(4.4). From (4.7), we obtain, by using Young's inequality, that

$$
\left\|\mathbf{u}_{\epsilon}\right\|_{L^{\infty}(0, T ; \mathbf{H})}^{2}+\sum_{i=1}^{N}\left\|D_{i} \mathbf{u}_{\epsilon}\right\|_{\mathbf{L}^{q_{i}\left(Q_{T}\right)}}^{q_{i}}+\epsilon\left\|\nabla \mathbf{u}_{\epsilon}\right\|_{\mathbf{L}^{\gamma}\left(Q_{T}\right)}^{\gamma} \leq C
$$


where, by the assumptions (3.8)-(3.9), $C$ is a positive constant and, very important, does not depend on $\epsilon$. The inequality (4.8) ensures that

$$
\begin{aligned}
& \left\|D_{i} \mathbf{u}_{\epsilon}\right\|_{\mathbf{L}^{q_{i}\left(Q_{T}\right)}} \leq C \quad \forall i \in\{1, \ldots, N\}, \\
& \left\|\mathbf{u}_{\epsilon}\right\|_{\mathrm{L}^{\infty}(0, T ; \mathbf{H})}^{2}+\sum_{i=1}^{N}\left\|\mathbf{u}_{\epsilon}\right\|_{\mathrm{L}^{q_{i}\left(0, T ; \mathbf{V}_{q_{i}}\right.}}^{q_{i}} \leq C, \\
& \left\|\left|D_{i} \mathbf{u}_{\epsilon}\right|^{q_{i}-2} D_{i} \mathbf{u}_{\epsilon}\right\|_{\mathbf{L}^{q_{i}^{\prime}\left(Q_{T}\right)}} \leq C \quad \text { for all } i \in\{1, \ldots, N\} .
\end{aligned}
$$

On the other hand, by using (4.10) and the imbedding (3.6), we can prove that

$$
\left\|\mathbf{u}_{\epsilon}\right\|_{\mathrm{L}^{\rho}\left(Q_{T}\right)} \leq C \quad \text { for } \quad \rho=2+\alpha-\frac{2 \alpha}{q_{a}^{*}} .
$$

As a consequence of (4.12),

$$
\left\|\mathbf{u}_{\epsilon} \otimes \mathbf{u}_{\epsilon}\right\|_{\mathbf{L}^{\frac{\rho}{2}\left(Q_{T}\right)}} \leq C .
$$

Note that the constants in (4.9)-(4.13) are distinct and do not depend on $\epsilon$. It should be stressed that the assumption (3.10) assures that $\rho>2$ and therefore $\mathbf{L}^{\frac{\rho}{2}}\left(Q_{T}\right)$ is a reflexive Banach space. Thus, from (4.9)-(4.13) and by means of reflexivity, there exists a sequence of positive numbers $\epsilon_{m}$ such that $\epsilon_{m} \longrightarrow 0$, as $m \rightarrow \infty$, and

$$
\begin{aligned}
& D_{i} \mathbf{u}_{\epsilon_{m}} \rightarrow D_{i} \mathbf{u} \quad \text { in } \mathbf{L}^{q_{i}}\left(Q_{T}\right), \quad \text { as } m \rightarrow \infty, \quad \text { for all } i \in\{1, \ldots, N\}, \\
& \left.\left|D_{i} \mathbf{u}_{\epsilon_{m}}\right|\right|^{q_{i}-2} D_{i} \mathbf{u}_{\epsilon_{m}} \rightarrow \mathbf{S}_{i} \quad \text { in } \mathbf{L}^{q_{i}^{\prime}}\left(Q_{T}\right), \quad \text { as } m \rightarrow \infty, \quad \text { for all } i \in\{1, \ldots, N\}, \\
& \mathbf{u}_{\epsilon_{m}} \rightarrow \mathbf{u} \text { in } \mathrm{L}^{\overrightarrow{\mathbf{q}}}\left(0, T ; \mathbf{V}_{\overrightarrow{\mathbf{q}}}\right), \quad \text { as } m \rightarrow \infty, \\
& \mathbf{u}_{\epsilon_{m}} \rightarrow \mathbf{u} \text { in } \mathbf{L}^{\rho}\left(Q_{T}\right), \quad \text { as } m \rightarrow \infty, \\
& \mathbf{u}_{\epsilon_{m}} \otimes \mathbf{u}_{\epsilon_{m}} \rightarrow \mathbf{G} \quad \text { in } \mathbf{L}^{\frac{\rho}{2}}\left(Q_{T}\right), \quad \text { as } m \rightarrow \infty .
\end{aligned}
$$

Moreover, it can be proved that

$$
\epsilon_{m}\left|\nabla \mathbf{u}_{\epsilon_{m}}\right|^{\gamma-2} \nabla \mathbf{u}_{\epsilon_{m}} \rightarrow \mathbf{0} \quad \text { in } \mathbf{L}^{\gamma^{\prime}}\left(Q_{T}\right), \quad \text { as } m \rightarrow \infty .
$$

Indeed, for $\varphi \in \mathrm{L}^{\gamma}\left(0, T ; \mathbf{V}_{\gamma}\right)$, we have by the application of Hölder's inequality and (4.8) that

$$
\begin{aligned}
& \left.\left|\int_{Q_{T}} \epsilon_{m}\right| \nabla \mathbf{u}_{\epsilon_{m}}\right|^{\gamma-2} \nabla \mathbf{u}_{\epsilon_{m}}: \nabla \varphi d \mathbf{x} d t \mid \leq \\
& C \epsilon_{m}^{\frac{1}{\gamma}}\left(\int_{Q_{T}} \epsilon_{m}\left|\nabla \mathbf{u}_{\epsilon_{m}}\right|^{\gamma} d \mathbf{x} d t\right)^{\frac{1}{\gamma^{\prime}}}\|\boldsymbol{\nabla} \varphi\|_{\mathbf{L}^{\gamma}\left(Q_{T}\right)} \leq C \epsilon_{m}^{\frac{1}{\gamma}} \longrightarrow 0, \text { as } m \rightarrow \infty .
\end{aligned}
$$

Then, using the convergence results (4.16)-(4.19), we can pass to the limit $\epsilon_{m} \longrightarrow 0$ in (4.6), with $\mathbf{u}_{\epsilon}$ replaced by $\mathbf{u}_{\epsilon_{m}}$, to obtain

$$
-\int_{Q_{T}} \mathbf{u} \cdot \varphi_{t} d \mathbf{x} d t+\sum_{i=1}^{N} \int_{Q_{T}} \mathbf{S}_{i} \cdot D_{i} \varphi d \mathbf{x} d t-\int_{Q_{T}}(\mathbf{G}+\mathbf{F}): \nabla \boldsymbol{\varphi} d \mathbf{x} d t=\int_{\Omega} \mathbf{u}_{0} \cdot \boldsymbol{\varphi}(0) d \mathbf{x}
$$

for all $\boldsymbol{\varphi} \in \mathbf{C}^{\infty}\left(Q_{T}\right)$, with $\operatorname{div} \varphi=0$ and $\operatorname{supp} \varphi \subset \subset \Omega \times[0, T)$. Now, using the identity (4.20) and proceeding analogously to $[28$, p. 123$]$, we can prove that

$$
\mathbf{u} \in \mathrm{C}_{\mathrm{w}}([0, T] ; \mathbf{H}) .
$$

The main difficulty here is to prove, from (4.20), that, for the distributional time derivative $\mathbf{u}_{t}$,

$$
\mathbf{u}_{t} \in \mathrm{L}^{\frac{N+1}{N}}\left(0, T ; \mathbf{Y}^{\prime}\right), \quad \mathbf{Y}:=\bigcap_{i=1}^{N} \mathbf{V}_{q_{i}} \cap \mathbf{V}_{\kappa} \cap \mathbf{H}, \quad \kappa:=\left(\frac{N+2}{N}\right)^{\prime} .
$$

To prove this we first observe that $\mathrm{L}^{\frac{N+1}{N}}\left(0, T ; \mathbf{Y}^{\prime}\right)$ denotes the dual space of $\mathrm{L}^{N+1}(0, T ; \mathbf{Y})$. Due to (4.15), we can prove that $D_{i} \mathbf{S}_{i} \in \mathrm{L} \frac{N+1}{N}\left(0, T ; \mathbf{V}_{q_{i}}^{\prime}\right)$ and due to the assumption (3.8), we can also prove that $\mathbf{F} \in$ $\mathrm{L}^{\frac{N+1}{N}}\left(0, T ; \mathbf{V}_{q_{i}}^{\prime}\right)$ for all $i \in\{1, \ldots, N\}$. That $\operatorname{div} \mathbf{G} \in \mathrm{L}^{\frac{N+1}{N}}\left(0, T ; \mathbf{V}_{\kappa}^{\prime}\right)$ follows exactly in the same way as it 
was done in [28]. Next, using (4.10) and the definition of the distributive time derivative $\frac{\partial \mathbf{u}_{\epsilon_{m}}}{\partial t}$, that is also in $\mathrm{L}^{\frac{N+1}{N}}\left(0, T ; \mathbf{Y}^{\prime}\right)$, we can prove analogously to [28] that for every $t \in[0, T]$

$$
\mathbf{u}_{\epsilon_{m}}(t) \rightarrow \mathbf{u}(t) \quad \text { in } \mathbf{H}, \quad \text { as } m \rightarrow \infty .
$$

Whence (4.21) holds, in particular $\mathbf{u}(0)=\mathbf{u}_{0}$ and, owe to this and to (4.3),

$$
\mathbf{u}_{\epsilon_{m}}(0)=\mathbf{u}(0) \text {. }
$$

\section{Convergence of the Approximated Convective term}

In this section, we shall prove that $\mathbf{G}=\mathbf{u} \otimes \mathbf{u}$. To do it so, we first consider a fixed but arbitrary domain $\omega$ for which we may well assume the existence of another domain $\omega^{\prime}$ such that

$$
\omega \subset \subset \omega^{\prime} \subset \subset \Omega: \quad \partial \omega \text { is Lipschitz-continuous and } \partial \omega^{\prime} \in \mathrm{C}^{2} .
$$

For these domains, we fix the notations $\omega_{T}:=\omega \times(0, T)$ and $\omega_{T}^{\prime}:=\omega^{\prime} \times(0, T)$. We start by observing that from (4.6) with $\mathbf{u}_{\epsilon_{m}}$ in the place of $\mathbf{u}_{\epsilon}$, and using the notations (3.8) and (3.11), we can write

$$
\begin{aligned}
& -\int_{\omega_{T}^{\prime}} \mathbf{u}_{\epsilon_{m}} \cdot \boldsymbol{\varphi}_{t} d \mathbf{x} d t+ \\
& \int_{\omega_{T}^{\prime}}\left(\sum_{i=1}^{N}\left|D_{i} \mathbf{u}_{\epsilon_{m}}\right|^{q_{i}-2} D_{i} \mathbf{u}_{\epsilon_{m}} \otimes \mathbf{e}_{i}-\mathbf{u}_{\epsilon_{m}} \otimes \mathbf{u}_{\epsilon_{m}}-\mathbf{F}\right): \nabla \boldsymbol{\varphi} d \mathbf{x} d t=0
\end{aligned}
$$

for all $\varphi \in \mathbf{C}_{0}^{\infty}\left(\omega_{T}^{\prime}\right)$, with $\operatorname{div} \varphi=0$. From the assumption (3.8) and the estimates (4.11) and (4.13), we verify that

$$
\sum_{i=1}^{N}\left|D_{i} \mathbf{u}_{\epsilon_{m}}\right|^{q_{i}-2} D_{i} \mathbf{u}_{\epsilon_{m}} \otimes \mathbf{e}_{i}-\mathbf{u}_{\epsilon_{m}} \otimes \mathbf{u}_{\epsilon_{m}}-\mathbf{F} \in \mathbf{L}^{r}\left(\omega_{T}^{\prime}\right),
$$

where

$$
1<r \leq \min \left\{\frac{\rho}{2}, \beta^{\prime}\right\}, \quad \text { with } \rho \text { given at }(3.6) \text {. }
$$

Observe that by assumption (3.10), $\rho>2$ and consequently $\min \left\{\frac{\rho}{2}, \beta^{\prime}\right\}>1$. Using (5.2)-(5.3), we can obtain, from (5.1), that the weak time derivative

$$
\mathbf{u}_{\epsilon_{m}}^{\prime} \in \mathrm{L}^{r}\left(0, T ; \mathbf{W}^{-1, r}\left(\omega^{\prime}\right)\right) .
$$

Now, we observe from (2.4) and since, by assumption (3.10), $q_{a}^{*}>\rho$, there always exists $\gamma>1$ such that the following Sobolev compact and continuous imbeddings hold

$$
\mathbf{W}_{0}^{1, \overrightarrow{\mathbf{q}}}\left(\omega^{\prime}\right) \hookrightarrow \hookrightarrow \mathbf{L}^{\gamma}\left(\omega^{\prime}\right) \hookrightarrow \mathbf{W}^{-1, r}\left(\omega^{\prime}\right), \quad\left(\left(r^{\prime}\right)^{*}\right)^{\prime} \leq \gamma<q_{a}^{*} .
$$

Then, using the Aubin-Lions compactness lemma (see [15, p. 58]), we obtain from (4.16) together with (5.4) and (5.5), and passing to a subsequence, that

$$
\mathbf{u}_{\epsilon_{m}} \longrightarrow \mathbf{u} \quad \text { in } \quad \mathrm{L}^{r}\left(0, T ; \mathbf{L}^{\gamma}\left(\omega^{\prime}\right)\right), \quad \text { as } \quad m \rightarrow \infty .
$$

Using parabolic interpolation, we obtain from (4.10) and (5.6) that

$$
\mathbf{u}_{\epsilon_{m}} \longrightarrow \mathbf{u} \quad \text { in } \quad \mathrm{L}^{s}\left(0, T ; \mathbf{L}^{\gamma}\left(\omega^{\prime}\right)\right) \quad \forall s: 1 \leq s<\infty, \quad \text { as } \quad m \rightarrow \infty .
$$

Since, from assumptions (3.10), $q_{a}^{*}>\rho>2$, we can choose $\gamma \in\left[\rho, q_{a}^{*}\right)$ such that, in view of (5.7),

$$
\mathbf{u}_{\epsilon_{m}} \longrightarrow \mathbf{u} \quad \text { in } \quad \mathbf{L}^{s}\left(\omega_{T}^{\prime},\right), \quad s=\rho, \quad \text { as } \quad m \rightarrow \infty,
$$

and in consequence

$$
\mathbf{u}_{\epsilon_{m}} \otimes \mathbf{u}_{\epsilon_{m}} \rightarrow \mathbf{u} \otimes \mathbf{u} \quad \text { in } \mathbf{L}^{\frac{\rho}{2}}\left(\omega_{T}^{\prime}\right), \quad \text { as } m \rightarrow \infty .
$$

From (4.18) and (5.9), we conclude that

$$
\mathbf{G}=\mathbf{u} \otimes \mathbf{u} .
$$




\section{Decomposition of the PREssure.}

First of all, we observe that by combining (4.20) with (5.1) and using (3.8) together with (4.22) and (5.10), we get

$$
\begin{aligned}
& -\int_{Q_{T}}\left(\mathbf{u}_{\epsilon_{m}}-\mathbf{u}\right) \cdot \boldsymbol{\varphi}_{t} d \mathbf{x} d t+\sum_{i=1}^{N} \int_{Q_{T}}\left[\left(\left.\left|D_{i} \mathbf{u}_{\epsilon_{m}}\right|\right|^{q_{i}-2} D_{i} \mathbf{u}_{\epsilon_{m}}-\mathbf{S}_{i}\right) \otimes \mathbf{e}_{i}\right]: \nabla \boldsymbol{\varphi} d \mathbf{x} d t= \\
& \int_{Q_{T}}\left(\mathbf{u}_{\epsilon_{m}} \otimes \mathbf{u}_{\epsilon_{m}}-\mathbf{u} \otimes \mathbf{u}\right): \nabla \boldsymbol{\varphi} d \mathbf{x} d t
\end{aligned}
$$

for all $\boldsymbol{\varphi} \in \mathbf{C}^{\infty}\left(Q_{T}\right)$, with $\operatorname{div} \varphi=0$ and $\operatorname{supp} \varphi \subset \subset \Omega \times[0, T)$. Now, the results (4.15), (4.21), (5.9) and (6.1) allow us to apply directly [28, Theorem 2.6] with the modifications introduced in [7, Theorem 2.2]. By these results, we can infer the existence of unique functions

$$
\begin{aligned}
& p_{\epsilon_{m}}^{1} \in \mathrm{L}^{q_{1}^{\prime}}\left(\omega_{T}^{\prime}\right), \ldots, p_{\epsilon_{m}}^{N} \in \mathrm{L}^{q_{N}^{\prime}}\left(\omega_{T}^{\prime}\right), \\
& p_{\epsilon_{m}}^{\otimes} \in \mathrm{L}^{\frac{\rho}{2}}\left(\omega_{T}^{\prime}\right), \\
& p_{\epsilon_{m}}^{h} \in \mathrm{C}_{\mathrm{w}}\left([0, T] ; \mathrm{W}^{1,2}\left(\omega^{\prime}\right)\right),
\end{aligned}
$$

with

$$
\triangle p_{\epsilon_{m}}^{h}=0 \quad \text { and } \quad p_{\epsilon_{m}}^{h}(0)=0
$$

and such that

$$
\begin{aligned}
& -\int_{\omega_{T}^{\prime}}\left(\mathbf{u}_{\epsilon_{m}}-\mathbf{u}\right) \cdot \boldsymbol{\varphi}_{t} d \mathbf{x} d t+\sum_{i=1}^{N} \int_{\omega_{T}^{\prime}}\left[\left(\left|D_{i} \mathbf{u}_{\epsilon_{m}}\right| q^{q_{i}-2} D_{i} \mathbf{u}_{\epsilon_{m}}-\mathbf{S}_{i}\right) \otimes \mathbf{e}_{i}\right]: \nabla \boldsymbol{\varphi} d \mathbf{x} d t= \\
& \int_{\omega_{T}^{\prime}}\left(\mathbf{u}_{\epsilon_{m}} \otimes \mathbf{u}_{\epsilon_{m}}-\mathbf{u} \otimes \mathbf{u}\right): \nabla \boldsymbol{\varphi} d \mathbf{x} d t+ \\
& \sum_{i=1}^{N} \int_{\omega_{T}^{\prime}} p_{\epsilon_{m}}^{i} \operatorname{div} \boldsymbol{\varphi} d \mathbf{x} d t+\int_{\omega_{T}^{\prime}} p_{\epsilon_{m}}^{\otimes} \operatorname{div} \boldsymbol{\varphi} d \mathbf{x} d t+\int_{\omega_{T}^{\prime}} \nabla p_{\epsilon_{m}}^{h} \boldsymbol{\varphi}_{t} d \mathbf{x} d t
\end{aligned}
$$

for all $\varphi \in \mathbf{C}^{\infty}\left(\omega_{T}^{\prime}\right)$ with supp $\varphi \subset \subset \omega^{\prime} \times[0, T)$. In addition, the following estimates also hold

$$
\begin{aligned}
& \left\|p_{\epsilon_{m}}^{i}\right\|_{L^{q_{i}^{\prime}\left(\omega_{T}^{\prime}\right)}} \leq C_{i}\left\|\left|D_{i} \mathbf{u}_{\epsilon_{m}}\right|^{q_{i}-2} D_{i} \mathbf{u}_{\epsilon_{m}}-\mathbf{S}_{i}\right\|_{\mathbf{L}^{q^{\prime}\left(\omega_{T}^{\prime}\right)}} \quad \forall i \in\{1, \ldots, N\}, \\
& \left\|p_{\epsilon_{m}}^{\otimes}\right\|_{\mathrm{L}^{\frac{\rho}{2}}\left(\omega_{T}^{\prime}\right)} \leq C_{\otimes}\left\|\mathbf{u}_{\epsilon_{m}} \otimes \mathbf{u}_{\epsilon_{m}}-\mathbf{u} \otimes \mathbf{u}\right\|_{\mathrm{L}^{\frac{\rho}{2}}\left(\omega_{T}^{\prime}\right)}, \\
& \left\|p_{\epsilon_{m}}^{h}(t)\right\|_{\mathrm{W}^{1,2}\left(\omega^{\prime}\right)} \leq C_{h}\left\|\mathbf{u}_{\epsilon_{m}}(t)-\mathbf{u}(t)\right\|_{\mathrm{L}^{2}\left(\omega^{\prime}\right)} \quad \forall t \in(0, T),
\end{aligned}
$$

where $C_{1}, \ldots, C_{N}, C_{\otimes}$ and $C_{h}$ are positive constants depending only on $N$, $\omega^{\prime}$ and on the correspondingly Lebesgue exponents. Then, using the local regularity theory together with (5.8) and (6.7), it can be proved (by the same reasoning used in $[7$, p.29]), that

$$
\left\|p_{\epsilon_{m}}^{h}\right\|_{\mathrm{L}^{s}\left(0, T ; \mathrm{W}^{2, s}(\omega)\right)} \longrightarrow 0 \quad \forall s: 1 \leq s<\infty, \quad \text { as } m \rightarrow \infty
$$

We now define

$$
\begin{aligned}
& \mathbf{v}_{\epsilon_{m}}:=\left(\mathbf{u}_{\epsilon_{m}}-\mathbf{u}+\nabla p_{\epsilon_{m}}^{h}\right) \chi_{\omega_{T}}, \\
& \mathbf{\Upsilon}_{\epsilon_{m}}^{i}:=\left(\mathbf{S}_{i}-\left|D_{i} \mathbf{u}_{\epsilon_{m}}\right|^{q_{i}-2} D_{i} \mathbf{u}_{\epsilon_{m}}\right) \otimes \mathbf{e}_{i}+p_{\epsilon_{m}}^{i} \mathbf{I}, \quad i \in\{1, \ldots, N\}, \\
& \mathbf{\Upsilon}_{\epsilon_{m}}^{\otimes}:=\mathbf{u}_{\epsilon_{m}} \otimes \mathbf{u}_{\epsilon_{m}}-\mathbf{u} \otimes \mathbf{u}+p_{\epsilon_{m}}^{\otimes} \mathbf{I}, \\
& \mathbf{\Upsilon}_{\epsilon_{m}}:=\sum_{i=1}^{N} \mathbf{\Upsilon}_{\epsilon_{m}}^{i}+\mathbf{\Upsilon}_{\epsilon_{m}}^{\otimes},
\end{aligned}
$$

where $\chi_{\omega_{T}}$ denotes the characteristic function of the set $\omega_{T}$. Observe that for (6.9), due to (5.8) and (6.8), we have

$$
\mathbf{v}_{\epsilon_{m}} \longrightarrow 0 \quad \text { in } \mathrm{L}^{\rho}\left(\omega_{T}\right), \quad \text { as } \quad m \rightarrow \infty
$$


Using (6.9)-(6.12), the integral identity (6.4) can be written in the form

$$
-\int_{\omega_{T}} \mathbf{v}_{\epsilon_{m}} \cdot \varphi_{t} d \mathbf{x} d t=\int_{\omega_{T}} \Upsilon_{\epsilon_{m}}: \nabla \varphi d \mathbf{x} d t
$$

for all $\varphi \in \mathbf{C}_{0}^{\infty}\left(\omega_{T}\right)$. From (6.14) it can be proved that for the distributive time derivative $\mathbf{v}_{\epsilon_{m}}^{\prime}$, we have

$$
\mathbf{v}_{\epsilon_{m}}^{\prime} \in \mathrm{L}^{r}\left(0, T ; \mathbf{W}^{-1, r}(\omega)\right),
$$

where $r$ satisfies to (5.3). In fact, due to (6.13), (4.15) and (6.5), (5.9) and (6.6), we respectively have

$$
\begin{aligned}
& \left\|\mathbf{v}_{\epsilon_{m}}\right\|_{\mathbf{L}^{\rho}\left(\omega_{T}\right)} \leq C, \\
& \left\|\mathbf{\Upsilon}_{\epsilon_{m}}^{i}\right\|_{\mathbf{L}^{q_{i}^{\prime}\left(\omega_{T}\right)}} \leq C \quad \forall i \in\{1, \ldots, N\}, \\
& \left\|\mathbf{\Upsilon}_{\epsilon_{m}}^{\otimes}\right\|_{\mathbf{L}^{\frac{\rho}{2}\left(\omega_{T}\right)}} \leq C,
\end{aligned}
$$

where $C$ denote distinct positive constants not depending on $m$. As a consequence of (6.16)-(6.18), we have

$$
\mathbf{\Upsilon}_{\epsilon_{m}} \in \mathbf{L}^{r}\left(\omega_{T}\right)
$$

and, consequently, $\operatorname{div} \Upsilon_{\epsilon_{m}} \in \mathrm{L}^{r}\left(0, T ; \mathbf{W}^{-1, r}(\omega)\right)$. Therefore, in view of (6.15) and (6.19), the integral identity (6.4) can also be written as

$$
\int_{0}^{T}\left\langle\mathbf{v}_{\epsilon_{m}}^{\prime}, \boldsymbol{\varphi}\right\rangle d t=\int_{\omega_{T}} \Upsilon_{\epsilon_{m}}: \nabla \boldsymbol{\varphi} d \mathbf{x} d t
$$

for all $\varphi \in \mathrm{L}^{r^{\prime}}\left(0, T ; \mathbf{W}_{0}^{1, r^{\prime}}(\omega)\right)$.

\section{Construction of a Lipschitz-COntinuous truncation}

In order to construct the Lipschitz truncation we shall work with in the rest of the proof, we consider the functions $\Upsilon_{\epsilon_{m}}^{i}$ and $\Upsilon_{\epsilon_{m}}^{\otimes}$ defined at (6.10)-(6.12) as being extended to $\mathbb{R}^{N+1}$ by zero. First of all, we observe that in view of (6.13),

$$
\mathbf{v}_{\epsilon_{m}} \longrightarrow 0 \quad \text { in } \mathbf{L}^{\rho}\left(\mathbb{R}^{N+1}\right), \quad \text { as } m \rightarrow \infty,
$$

Using (4.16), (6.7) and (6.9) on one hand, and using (6.17) on the other, we have for every $i \in\{1, \ldots, N\}$

$$
\begin{gathered}
\left\|D_{i} \mathbf{v}_{\epsilon_{m}}\right\|_{\mathbf{L}^{q_{i}}\left(\mathbb{R}^{N+1}\right)} \leq C, \\
\left\|\mathbf{\Upsilon}_{\epsilon_{m}}^{i}\right\|_{\mathbf{L}^{q_{i}^{\prime}\left(\mathbb{R}^{N+1}\right)}} \leq C,
\end{gathered}
$$

for distinct positive constants $C$ not depending on $m$. Moreover, (5.9) together with (6.6), justify that

$$
\mathbf{\Upsilon}_{\epsilon_{m}}^{\otimes} \longrightarrow 0 \quad \text { in } \mathbf{L}^{\frac{\rho}{2}}\left(\mathbb{R}^{N+1}\right), \quad \text { as } m \rightarrow \infty \text {. }
$$

Let us now set

$$
\begin{aligned}
& f_{\epsilon_{m}}:=\mathcal{M}^{*}\left(\left|\mathbf{v}_{\epsilon_{m}}\right|\right), \\
& g_{\epsilon_{m}}^{i}:=\mathcal{M}^{*}\left(\left|D_{i} \mathbf{v}_{\epsilon_{m}}\right|\right)+\left(\mathcal{M}^{*}\left(\left|\mathbf{\Upsilon}_{\epsilon_{m}}^{i}\right|\right)\right)^{\frac{1}{q_{i}-1}}, \quad i \in\{1, \ldots, N\}, \\
& h_{\epsilon_{m}}^{i}:=\left(\mathcal{M}^{*}\left(\left|\mathbf{\Upsilon}_{\epsilon_{m}}^{\otimes}\right|\right)\right)^{\frac{1}{q_{i}-1}}, \quad i \in\{1, \ldots, N\},
\end{aligned}
$$

where $\mathcal{M}^{*}:=\mathcal{M}_{t} \circ \mathcal{M}_{\mathbf{x}}$. Here $\mathcal{M}_{t}$ and $\mathcal{M}_{\mathbf{x}}$ denote the Hardy-Littlewood maximal operators, which are defined, for some function $f \in \mathrm{L}^{p}\left(\mathbb{R}^{N+1}\right)$ with $1<p<\infty$, respectively by

$$
\begin{aligned}
& \mathcal{M}_{t}(f)(\mathbf{x}, t):=\sup _{0<r<\infty} \frac{1}{2 r} \int_{t-r}^{t+r}|f(\mathbf{x}, s)| d s, \\
& \mathcal{M}_{\mathbf{x}}(f)(\mathbf{x}, t):=\sup _{0<R<\infty} \frac{1}{\mathcal{L}_{N}\left(B_{R}(\mathbf{x})\right)} \int_{B_{R}(\mathbf{x})}|f(\mathbf{y}, s)| d \mathbf{y},
\end{aligned}
$$


where $B_{R}(\mathbf{x})$ denotes the ball of $\mathbb{R}^{N}$ centered at $\mathbf{x}$ with radius $R>0$, and $\mathcal{L}_{N}$ stands for the $N$-dimensional Lebesgue measure. Then due to the boundedness of the operator $\mathcal{M}^{*}$ from $\mathbf{L}^{p}\left(\mathbb{R}^{N+1}\right)$ into $\mathbf{L}^{p}\left(\mathbb{R}^{N+1}\right)$ for any $p>1$ (see e.g. Stein [25, Theorem I.1.1]), it can be proved that

$$
\begin{aligned}
& \left\|f_{\epsilon_{m}}\right\|_{\mathbf{L}^{\rho}\left(\mathbb{R}^{N+1}\right)} \longrightarrow 0, \quad \text { as } m \rightarrow \infty, \\
& \left\|g_{\epsilon_{m}}^{i}\right\|_{\mathbf{L}^{q_{i}\left(\mathbb{R}^{N+1}\right)}} \leq C \quad \forall i \in\{1, \ldots, N\}, \\
& \left\|h_{\epsilon_{m}}^{i}\right\|_{\mathbf{L}^{\frac{\rho}{2}}\left(\mathbb{R}^{N+1}\right)} \longrightarrow 0 \quad \forall i \in\{1, \ldots, N\}, \quad \text { as } m \rightarrow \infty,
\end{aligned}
$$

for some positive constant $C$ not depending on $m$. To prove (7.4) we have used (5.8) and (6.8), to prove (7.5) we used (4.16), (6.7) and (6.17), whereas to prove (7.6) we used (5.9). Next, by the reasoning of [7, p. 31] (see also [2, Lemma 5.2]), we can infer, for every $i \in\{1, \ldots, N\}$ and for every $j, m \in \mathbb{N}$, the existence of $\lambda_{m, j} \in\left[2^{2^{j}+1}, 2^{2^{j+1}}\right]$ such that

$$
\begin{aligned}
& \mathcal{L}_{N+1}\left(G_{m, j}^{i}\right) \leq C 2^{-j} \lambda_{m, j}^{-q_{i}}\left\|g_{\epsilon_{m}}^{i}\right\|_{\mathbf{L}^{q_{i}}\left(\mathbb{R}^{N+1}\right)}, \quad G_{m, j}^{i}:=\left\{(\mathbf{x}, t) \in \mathbb{R}^{N+1}:\left|g_{\epsilon_{m}}^{i}\right|>\lambda_{m, j}\right\}, \\
& \mathcal{L}_{N+1}\left(H_{m, j}^{i}\right) \leq C 2^{-j} \lambda_{m, j}^{\left(1-q_{i}\right) \frac{\rho}{2}}\left\|h_{\epsilon_{m}}^{i}\right\|_{\mathbf{L}^{\frac{\rho}{2}}\left(\mathbb{R}^{N+1}\right)}, \quad H_{m, j}^{i}:=\left\{(\mathbf{x}, t) \in \mathbb{R}^{N+1}:\left|h_{\epsilon_{m}}^{i}\right|>\lambda_{m, j}\right\} .
\end{aligned}
$$

On the other hand, for every $j, m \in \mathbb{N}$, we also have, by virtue of (7.1) and (7.4),

$$
\mathcal{L}_{N+1}\left(F_{m, j}\right) \leq C\left\|f_{\epsilon_{m}}\right\|_{\mathbf{L}^{\rho}\left(\mathbb{R}^{N+1}\right)}, \quad F_{m, j}:=\left\{(\mathbf{x}, t) \in \mathbb{R}^{N+1}:\left|f_{\epsilon_{m}}\right|>1\right\} .
$$

Gathering (7.5) and (7.7), we have

$$
\mathcal{L}_{N+1}\left(G_{m, j}^{i}\right) \leq C 2^{-j} \lambda_{m, j}^{-q_{i}} \quad \forall i \in\{1, \ldots, N\}
$$

Moreover, we also have

$$
G_{m, j}^{i} \supset\left\{\mathcal{M}^{*}\left(\left|D_{i} \mathbf{v}_{\epsilon_{m}}\right|\right)>\lambda_{m, j}\right\} \cup\left\{\mathcal{M}^{*}\left(\left|\Upsilon_{\epsilon_{m}}^{i}\right|\right)>\lambda_{m, j}^{q_{i}-1}\right\} \quad \forall i \in\{1, \ldots, N\} .
$$

Now, since $\lambda_{m, j} \in\left[2^{2^{j}+1}, 2^{2^{j+1}}\right]$, we observe that (7.4) and (7.9) on the one hand and (7.6) and (7.8) on the other, imply, respectively, that

$$
\begin{aligned}
& \limsup _{m \rightarrow \infty} \mathcal{L}_{N+1}\left(F_{m, j}\right)=0, \\
& \limsup _{m \rightarrow \infty} \mathcal{L}_{N+1}\left(H_{m, j}^{i}\right)=0 \quad \forall i \in\{1, \ldots, N\} .
\end{aligned}
$$

On the other hand, since $\mathcal{M}^{*}$ is subadditive (see e.g. Stein [25]), we get from the definitions of $G_{m, j}^{i}$ and $H_{m, j}^{i}$ (see (7.7)-(7.8)) that, for each $i \in\{1, \ldots, N\}$,

$$
G_{m, j}^{i} \cup H_{m, j}^{i} \supset \mathcal{O}^{i}
$$

where

$$
\begin{aligned}
& \mathcal{O}^{i}:=\left\{(\mathbf{x}, t) \in \mathbb{R}^{N+1}: \mathcal{M}^{*}\left(\left|D_{i} \mathbf{v}_{\epsilon_{m}}\right|\right)+\varrho_{m, j}^{i}\left(\mathcal{M}^{*}\left(\left|\mathbf{\Upsilon}_{\epsilon_{m}}^{i}\right|\right)+\mathcal{M}^{*}\left(\left|\mathbf{\Upsilon}_{\epsilon_{m}}^{\otimes}\right|\right)\right)>3 \lambda_{m, j}\right\}, \\
& \varrho_{m, j}^{i}:=\lambda_{m, j}^{2-q_{i}} .
\end{aligned}
$$

For each $i \in\{1, \ldots, N\}$, we set

$$
E_{m, j}^{i}:=\left(F_{m, j} \cup G_{m, j}^{i} \cup H_{m, j}^{i}\right) \cap \omega_{T},
$$

Then, we can readily see that due to (7.10) and (7.11)-(7.12), we have

$$
\limsup _{m \rightarrow \infty} \mathcal{L}_{N+1}\left(E_{m, j}^{i}\right) \leq C 2^{-j} \limsup _{m \rightarrow \infty} \lambda_{m, j}^{-q_{i}}, \quad i \in\{1, \ldots, N\} .
$$

Moreover, due to (7.13), we have, for each $i \in\{1, \ldots, N\}$,

$$
\left(\mathcal{O}^{i} \cup \mathcal{U}\right) \cap \omega_{T} \subset E_{m, j}^{i} \subset \omega_{T},
$$

where here $\mathcal{U}$ is the set $F_{m, j}$ defined in (7.9).

We are now in conditions to define the truncation we shall consider here. For each $i \in\{1, \ldots, N\}$, we consider the following Whitney covering of $E_{m, j}^{i}$ formed by the cubes

$$
Q_{r_{n}}^{\varrho_{m, j}^{i}} \equiv Q_{r_{n}}^{\varrho_{m, j}^{i}}\left(\mathbf{x}_{n}, t_{n}\right):=\left\{(\mathbf{y}, s) \in \mathbb{R}^{N+1}: d_{\varrho_{m, j}^{i}}\left(\left(\mathbf{x}_{n}, t_{n}\right),(\mathbf{y}, s)\right)<r_{n}\right\}
$$


where $r_{n}>0, n \in \mathbb{N}$ and $d_{\varrho_{m, j}^{i}}$ is the metric defined by

$$
d_{\varrho_{m, j}^{i}}\left(\left(\mathbf{x}_{n}, t_{n}\right),(\mathbf{y}, s)\right):=\max \left\{\left|\mathbf{y}-\mathbf{x}_{n}\right|, \frac{1}{\varrho_{m, j}^{i}} \sqrt{\left|s-t_{n}\right|}\right\},
$$

and $\left\{\psi_{n}^{i}: n \in \mathbb{N}\right\}$ is the associated partition of unity (see e.g. Stein [25]). Following [7, Section 3], we define, for each $i \in\{1, \ldots, N\}$, the Lipschitz truncation of $\mathbf{v}_{\epsilon_{m}}$ subordinated to the Whitney covering (7.17)-(7.18) by

$$
\mathcal{T}_{m, j}^{i}\left(\mathbf{v}_{\epsilon_{m}}\right):=\left\{\begin{array}{llr}
\mathbf{v}_{\epsilon_{m}} & \text { in } & \omega_{T} \backslash E_{m, j}^{i} \\
\sum_{n=1}^{\infty} \psi_{n}^{i} \mathbf{v}_{\epsilon_{m}} \chi_{Q_{r_{n}}^{e_{m, j}^{i}}} & \text { in } & E_{m, j}^{i},
\end{array}\right.
$$

where $\chi_{Q_{r_{n}}^{e_{m, j}^{i}}}$ denotes the characteristic function of the set $Q_{r_{n}}^{\varrho_{m, j}^{i}}$. Here, we observe that for $i \neq k$ the irregularity sets $E_{m, j}^{i}$ and $E_{m, j}^{k}$ are not necessarily the same. Therefore, we may have $\mathcal{T}_{m, j}^{i}\left(\mathbf{v}_{\epsilon_{m}}\right)=\mathbf{v}_{\epsilon_{m}}$ in all $E_{m, j}^{k}$ or just in part of it, if $E_{m, j}^{i} \cap E_{m, j}^{k}=\emptyset$ or $E_{m, j}^{i} \cap E_{m, j}^{k} \neq \emptyset$, respectively. Taking this into account and defining

$$
E_{m, j}:=\bigcap_{i=1}^{N} E_{m, j}^{i},
$$

with the help of all $\mathcal{T}_{m, j}^{i}$ we can build the following Lipschitz truncation of $\mathbf{v}_{\epsilon_{m}}$

$$
\mathcal{T}_{m, j}\left(\mathbf{v}_{\epsilon_{m}}\right):= \begin{cases}\mathbf{v}_{\epsilon_{m}} & \text { in } \omega_{T} \backslash E_{m, j} \\ \mathcal{T}_{m, j}^{i}\left(\mathbf{v}_{\epsilon_{m}}\right) & \text { in } E_{m, j}^{k} \backslash\left(E_{m, j}^{k} \cap E_{m, j}^{i}\right), \quad \text { if } E_{m, j}^{k} \cap E_{m, j}^{i} \neq \emptyset \\ \sum_{i=1}^{N} \sum_{n=1}^{\infty} \psi_{n}^{i} \mathbf{v}_{\epsilon_{m}} \chi_{Q_{r_{n}}^{e_{m, j}^{i}}} & \text { in } E_{m, j},\end{cases}
$$

Note that if $E_{m, j}^{k} \cap E_{m, j}^{i} \neq \emptyset$, than $\mathcal{T}_{m, j}^{i}\left(\mathbf{v}_{\epsilon_{m}}\right)=\mathbf{v}_{\epsilon_{m}}$ in $E_{m, j}^{k} \backslash\left(E_{m, j}^{k} \cap E_{m, j}^{i}\right)$. Now, let $\xi \in \mathrm{C}_{0}^{\infty}\left(\omega_{T}\right)$ be a fixed cut-off function such that $0 \leq \xi \leq 1$ in $\omega_{T}$ and let us consider the following admissible test function

$$
\phi_{m, j}:=\xi \mathcal{T}_{m, j}\left(\mathbf{v}_{\epsilon_{m}}\right) .
$$

In order to establish the main properties of the Lipschitz truncation (7.20) we are interested in, let us set

$$
\omega_{T}^{\xi}:=\operatorname{supp} \xi, \quad \xi \text { is the cut-off function of }(7.21) .
$$

Note that $\omega_{T}^{\xi}$ is strictly contained in $\omega_{T}$, because $0 \leq \xi \leq 1$ in $\omega_{T}$. Let also $\mathbf{C}_{d_{\varrho_{m, j}}^{0,1}}\left(\omega_{T}^{\xi}\right)$ be the space of Lipschitz-continuous functions with respect to the metric (7.18). From the definition of $\mathbf{v}_{\epsilon_{m}}$ (see (6.9)), using (4.16) together with (6.6), we can prove that

$$
\mathbf{v}_{\epsilon_{m}} \in \mathrm{L}^{\infty}\left(0, T ; \mathbf{L}^{2}(\omega)\right) \cap \mathrm{L}^{\overrightarrow{\mathbf{q}}}\left(0, T ; \mathbf{V}_{\overrightarrow{\mathbf{q}}}\right) .
$$

Then, owing to (7.11)-(7.16), we can apply directly [7, Theorem 3.9, (i)-(iii)] to obtain:

$$
\mathcal{T}_{m, j}\left(\mathbf{v}_{\epsilon_{m}}\right) \in \mathbf{C}_{d_{\varrho_{m, j}}^{0,1}}\left(\omega_{T}^{\xi}\right),
$$

with the norm depending on $N, \omega_{T}^{\xi},\left\|\mathbf{v}_{\epsilon_{m}}\right\|_{\mathbf{L}^{1}\left(E_{m, j}\right)},\left\|\mathbf{v}_{\epsilon_{m}}\right\|_{\mathbf{L}^{1}\left(\widetilde{\omega}_{T}\right)}$, where $\omega_{T}^{\xi} \subset \subset \widetilde{\omega}_{T} \subset \subset \omega_{T}$;

$$
\begin{aligned}
& \left\|\boldsymbol{\nabla}_{m, j}\left(\mathbf{v}_{\epsilon_{m}}\right)\right\|_{\mathbf{L}^{\infty}\left(\omega_{T}^{\xi}\right)} \leq C\left(\lambda_{m, j}+\varrho_{m, j}^{-1} \delta_{d_{\varrho_{m, j}, \omega_{T}^{\xi}}^{-N-3}}^{-\mathbf{v}_{\epsilon_{m}}} \|_{\mathbf{L}^{1}\left(E_{m, j}\right)}\right), \\
& \left\|\boldsymbol{T}_{m, j}\left(\mathbf{v}_{\epsilon_{m}}\right)\right\|_{\mathbf{L}^{\infty}\left(\omega_{T}^{\xi}\right)} \leq C\left(1+\varrho_{m, j}^{-1} \delta_{d_{\varrho_{m, j}, \omega_{T}^{\xi}}^{-N-2}}\left\|\mathbf{v}_{\epsilon_{m}}\right\|_{\mathbf{L}^{1}\left(E_{m, j}\right)}\right)
\end{aligned}
$$

where the constants denoted by $C$ depend only on $N$, and

$$
\delta_{d_{\varrho_{m, j}, \omega_{T}^{\xi}}}:=d_{\varrho_{m, j}}\left(\omega_{T}^{\xi}, \omega_{T}\right)>0 \quad \text { due to }(7.22)
$$




$$
\begin{aligned}
& \left\|\mathcal{T}_{m, j}^{\prime}\left(\mathbf{v}_{\epsilon_{m}}\right) \cdot\left(\mathcal{T}_{m, j}\left(\mathbf{v}_{\epsilon_{m}}\right)-\mathbf{v}_{\epsilon_{m}}\right)\right\|_{\mathbf{L}^{1}\left(\omega_{T}^{\xi} \cap E_{m, j}\right)} \leq \\
& C \varrho_{m, j}^{-1} \mathcal{L}_{N+1}\left(E_{m, j}\right)\left(\lambda_{m, j}+\varrho_{m, j}^{-1} \delta_{d_{\varrho_{m, j}}-N-\omega_{T}^{\xi}}^{-3}\left\|\mathbf{v}_{\epsilon_{m}}\right\|_{\mathbf{L}^{1}\left(E_{m, j}\right)}\right)^{2},
\end{aligned}
$$

where the constant $C$ depends only on $N$. In addition,

$$
\left\|\mathcal{T}_{m, j}\left(\mathbf{v}_{\epsilon_{m}}\right)\right\|_{\mathbf{L}^{s}\left(\omega_{T}\right)} \leq C\left\|\mathbf{v}_{\epsilon_{m}}\right\|_{\mathbf{L}^{s}\left(\omega_{T}\right)} \quad \forall s: 1 \leq s \leq \infty,
$$

where $C$ depends only on $N$ (see [7, Lemma 3.5] and [25, Section VI.3]).

\section{Convergence of the Anisotropic Diffusion term}

Observing that, by virtue of (7.23)-(7.25) and of the definition of $\xi$, our admissible test function $\phi_{m, j} \in$ $\mathrm{L}^{r^{\prime}}\left(0, T ; \mathbf{W}_{0}^{1, r^{\prime}}(\omega)\right)$, then, from (6.15) and (6.20), we infer that

$$
\int_{0}^{T}\left\langle\mathbf{v}_{\epsilon_{m}}^{\prime}(t), \phi_{m, j}(t)\right\rangle d t=\int_{\omega_{T}} \mathbf{\Upsilon}_{\epsilon_{m}}: \nabla \boldsymbol{\phi}_{m, j} d \mathbf{x} d t
$$

On the other hand, owing to (7.11)-(7.16) and, in addition, to (6.19), we can apply [7, Theorem 3.9, (iv)] to prove that for every $\xi \in \mathrm{C}_{0}^{\infty}\left(\omega_{T}\right)$

$$
\begin{aligned}
\int_{0}^{T}\left\langle\mathbf{v}_{\epsilon_{m}}^{\prime}(t), \phi_{m, j}(t)\right\rangle d t= & \frac{1}{2} \int_{\omega_{T}}\left(\left|\mathcal{T}_{m, j}\left(\mathbf{v}_{\epsilon_{m}}\right)\right|^{2}-2 \mathbf{v}_{\epsilon_{m}} \cdot \mathcal{T}_{m, j}\left(\mathbf{v}_{\epsilon_{m}}\right)\right) \xi^{\prime} d \mathbf{x} d t \\
& +\int_{E_{m, j}} \mathcal{T}_{m, j}^{\prime}\left(\mathbf{v}_{\epsilon_{m}}\right) \cdot\left(\mathcal{T}_{m, j}\left(\mathbf{v}_{\epsilon_{m}}\right)-\mathbf{v}_{\epsilon_{m}}\right) \xi d \mathbf{x} d t
\end{aligned}
$$

Now, gathering (6.20) and (8.1), and expanding the notations (6.10)-(6.11), we obtain

$$
\begin{aligned}
& \sum_{i=1}^{N} \int_{\omega_{T}}\left(\left.\left|D_{i} \mathbf{u}_{\epsilon_{m}}\right|\right|^{q_{i}-2} D_{i} \mathbf{u}_{\epsilon_{m}}-\mathbf{S}_{i}\right) \otimes \mathbf{e}_{i}: \nabla \mathcal{T}_{m, j}\left(\mathbf{v}_{\epsilon_{m}}\right) \xi d \mathbf{x} d t= \\
& \sum_{i=1}^{N} \int_{\omega_{T}}\left(\mathbf{S}_{i}-\left|D_{i} \mathbf{u}_{\epsilon_{m}}\right|^{q_{i}-2} D_{i} \mathbf{u}_{\epsilon_{m}}\right) \otimes \mathbf{e}_{i}: \mathcal{T}_{m, j}\left(\mathbf{v}_{\epsilon_{m}}\right) \otimes \nabla \xi d \mathbf{x} d t \\
& +\int_{\omega_{T}}\left(\mathbf{u}_{\epsilon_{m}} \otimes \mathbf{u}_{\epsilon_{m}}-\mathbf{u} \otimes \mathbf{u}\right): \nabla\left(\mathcal{T}_{m, j}\left(\mathbf{v}_{\epsilon_{m}}\right) \xi\right) d \mathbf{x} d t \\
& +\sum_{i=1}^{N} \int_{\omega_{T}} p_{\epsilon_{m}}^{i} \mathcal{T}_{m, j}\left(\mathbf{v}_{\epsilon_{m}}\right) \cdot \nabla \xi d \mathbf{x} d t \\
& +\sum_{i=1}^{N} \int_{\omega_{T}} p_{\epsilon_{m}}^{i} \operatorname{div}\left(\mathcal{T}_{m, j}\left(\mathbf{v}_{\epsilon_{m}}\right)\right) \xi d \mathbf{x} d t \\
& +\int_{\omega_{T}} p_{\epsilon_{m}}^{\otimes} \operatorname{div}\left(\mathcal{T}_{m, j}\left(\mathbf{v}_{\epsilon_{m}}\right) \xi\right) d \mathbf{x} d t \\
& +\frac{1}{2} \int_{\omega_{T}}\left(2 \mathbf{v}_{\epsilon_{m}} \cdot \mathcal{T}_{m, j}\left(\mathbf{v}_{\epsilon_{m}}\right)-\left|\mathcal{T}_{m, j}\left(\mathbf{v}_{\epsilon_{m}}\right)\right|^{2}\right) \xi^{\prime} d \mathbf{x} d t \\
& +\int_{E_{m, j}} \mathcal{T}_{m, j}^{\prime}\left(\mathbf{v}_{\epsilon_{m}}\right) \cdot\left(\mathbf{v}_{\epsilon_{m}}-\mathcal{T}_{m, j}\left(\mathbf{v}_{\epsilon_{m}}\right)\right) \xi d \mathbf{x} d t \\
& :=\sum_{i=1}^{N} J_{1}^{i}+J_{2}+\sum_{i=1}^{N} J_{3}^{i}+\sum_{i=1}^{N} J_{4}^{i}+J_{5}+J_{6}+J_{7}
\end{aligned}
$$

We claim that, for a fixed $j$,

$$
\limsup _{m \rightarrow \infty} \sum_{i=1}^{N}\left|\int_{\omega_{T}}\left(\left|D_{i} \mathbf{u}_{\epsilon_{m}}\right|^{q_{i}-2} D_{i} \mathbf{u}_{\epsilon_{m}}-\mathbf{S}_{i}\right) \otimes \mathbf{e}_{i}: \nabla \mathcal{T}_{m, j}\left(\mathbf{v}_{\epsilon_{m}}\right) \xi d \mathbf{x} d t\right| \leq C 2^{-\frac{j}{2}}
$$

To prove this, we will carry out the passage to the limit $m \rightarrow \infty$ in all absolute values $\left|J_{l}\right|, l=1, \ldots, 7$. 
- $\lim \sup _{m \rightarrow \infty} \sum_{i=1}^{N}\left(\left|J_{1}^{i}\right|+\left|J_{3}^{i}\right|\right)=0$. Due to (4.15) and (6.2), $\left(\mathbf{S}_{i}-\left|D_{i} \mathbf{u}_{\epsilon_{m}}\right|^{q_{i}-2} D_{i} \mathbf{u}_{\epsilon_{m}}\right) \otimes \mathbf{e}_{i}$ and $p_{\epsilon_{m}}^{i}$ are uniformly bounded in $\mathbf{L}^{q_{i}^{\prime}}\left(\omega_{T}\right)$ for all $i \in\{1, \ldots, N\}$. Then, using Hölder's inequality and (7.28), led us to

$$
\left|J_{1}^{i}\right|+\left|J_{3}^{i}\right| \leq C_{1}\left\|\mathcal{T}_{m, j}\left(\mathbf{v}_{\epsilon_{m}}\right)\right\|_{\mathbf{L}^{q_{i}\left(\omega_{T}\right)}} \leq C_{2}\left\|\mathbf{v}_{\epsilon_{m}}\right\|_{\mathbf{L}^{q_{i}\left(\omega_{T}\right)}} \leq C_{3}\left\|\mathbf{v}_{\epsilon_{m}}\right\|_{\mathbf{L}^{\beta}\left(\omega_{T}\right)}
$$

for all $i \in\{1, \ldots, N\}$. The assertion follows by the application of (5.7) with $s=\gamma=\beta$ and (6.8) with $s=\beta$, and observing that, from assumption (3.7), $\beta<q_{a}^{*}$.

- $\lim \sup _{m \rightarrow \infty}\left(\left|J_{2}\right|+\left|J_{5}\right|\right)=0$. In fact,

$$
\begin{aligned}
\left|J_{2}\right|+\left|J_{5}\right| \leq & \left\|\mathbf{u} \otimes \mathbf{u}-\mathbf{u}_{\epsilon_{m}} \otimes \mathbf{u}_{\epsilon_{m}}\right\|_{\mathbf{L}^{1}\left(\omega_{T}\right)}\left\|\boldsymbol{\nabla}\left(\mathcal{T}_{m, j}\left(\mathbf{v}_{\epsilon_{m}}\right) \xi\right)\right\|_{\mathbf{L}^{\infty}\left(\omega_{T}^{\xi}\right)}+ \\
& \left\|p_{\epsilon_{m}}^{\otimes}\right\|_{\mathbf{L}^{1}\left(\omega_{T}\right)}\left\|\operatorname{div}\left(\mathcal{T}_{m, j}\left(\mathbf{v}_{\epsilon_{m}}\right) \xi\right)\right\|_{\mathbf{L}^{\infty}\left(\omega_{T}^{\xi}\right)} .
\end{aligned}
$$

Then, due to (5.9) and (6.6), $\lim \sup _{m \rightarrow \infty}\left(\left|J_{2}\right|+\left|J_{5}\right|\right)=0$ if, for a fixed $j \in \mathbb{N}$, both second multiplying terms on the right-hand side of the above inequality are uniformly bounded with respect to $m$. This is true, because, according to (7.24) and (7.25), we get

$$
\begin{aligned}
& \left\|\boldsymbol{\nabla}\left(\mathcal{T}_{m, j}\left(\mathbf{v}_{\epsilon_{m}}\right) \xi\right)\right\|_{\mathbf{L}^{\infty}\left(\omega_{T}^{\xi}\right)} \leq \\
& \left\|\boldsymbol{\nabla} \mathcal{T}_{m, j}\left(\mathbf{v}_{\epsilon_{m}}\right)\right\|_{\mathbf{L}^{\infty}\left(\omega_{T}^{\xi}\right)}+C\left\|\boldsymbol{T}_{m, j}\left(\mathbf{v}_{\epsilon_{m}}\right)\right\|_{\mathbf{L}^{\infty}\left(\omega_{T}^{\xi}\right)} \leq \\
& C_{1}\left(\lambda_{m, j}+\varrho_{m, j}^{-1} \frac{\left\|\mathbf{v}_{\epsilon_{m}}\right\|_{\mathbf{L}^{1}\left(E_{m, j}\right)}}{\delta_{d_{\varrho_{m, j}}^{N+3} \omega_{T}^{\xi}}^{N+3}}\right)+C_{2}\left(1+\varrho_{m, j}^{-1} \frac{\left\|\mathbf{v}_{\epsilon_{m}}\right\|_{\mathbf{L}^{1}\left(E_{m, j}\right)}}{\delta_{d_{\varrho_{m, j}}+\omega_{T}^{\xi}}^{\xi}}\right) .
\end{aligned}
$$

Now, observe that from (6.16), $\mathbf{v}_{\epsilon_{m}}$ is uniformly bounded in $\mathbf{L}^{1}\left(E_{m, j}\right)$. Moreover, (7.18) and (7.26) implies that

$$
\inf _{m \in \mathbb{N}} \delta_{d, \omega_{T}^{\xi}}>0
$$

On the other hand, for a fixed $j \in \mathbb{N}$, the sequence $\lambda_{m, j}$ lies in the interval $\left[2^{2^{j}+1}, 2^{2^{j+1}}\right]$ and therefore $\varrho_{m, j}^{-1}$ is also uniformly bounded (from above) with respect to $m$.

- $\lim \sup _{m \rightarrow \infty} \sum_{i=1}^{N}\left|J_{4}^{i}\right| \leq C 2^{-\frac{j}{\beta}}$. By the definition of the Lipschitz truncation (see (7.20)) together with the fact that $\operatorname{div} \mathbf{v}_{\epsilon_{m}}=0$ (see (6.9) and (6.3)), we can write for each $i \in\{1, \ldots, N\}$

$$
J_{4}^{i}=\int_{\omega_{T}^{\xi} \cap E_{m, j}} p_{\epsilon_{m}}^{i} \operatorname{div} \mathcal{T}_{m, j}\left(\mathbf{v}_{\epsilon_{m}}\right) d \mathbf{x} d t \quad \forall i \in\{1, \ldots, N\} .
$$

Using Hölder's inequality, (6.5), (4.15) and (7.22) together with the same reasoning of the previous bullet, we have

$$
\begin{aligned}
\limsup _{m \rightarrow \infty}\left|J_{4}^{i}\right| & \leq C \limsup _{m \rightarrow \infty}\left\|\nabla \mathcal{T}_{m, j}\left(\mathbf{v}_{\epsilon_{m}}\right)\right\|_{\mathbf{L}^{q_{i}}\left(\omega_{T}^{\xi} \cap E_{m, j}\right)} \\
& \leq C \limsup _{m \rightarrow \infty}\left[\mathcal{L}_{N+1}\left(E_{m, j}\right)^{\frac{1}{q_{i}}}\left(\lambda_{m, j}+\varrho_{m, j}^{-1} \delta_{d_{\varrho_{m, j}}, \omega_{T}^{\xi}}^{-N-3}\left\|\mathbf{v}_{\epsilon_{m}}\right\|_{\mathbf{L}^{1}\left(E_{m, j}\right)}\right)\right] \\
& \leq C \limsup _{m \rightarrow \infty}\left(\mathcal{L}_{N+1}\left(E_{m, j}\right)^{\frac{1}{q_{i}}} \lambda_{m, j}\right) \leq C \limsup _{m \rightarrow \infty}\left(\mathcal{L}_{N+1}\left(E_{m, j}^{i}\right)^{\frac{1}{q_{i}}} \lambda_{m, j}\right)
\end{aligned}
$$

Then, by the definition of $E_{m, j}$ (see (7.19) and (7.14)) and (7.15) together with (7.2) and (7.3), we get the desired result.

- $\lim \sup _{m \rightarrow \infty}\left|J_{6}\right|=0$. By Cauchy-Schwarz's inequality and (7.28),

$$
\left|J_{6}\right| \leq C\left\|\mathbf{v}_{\epsilon_{m}}\right\|_{\mathbf{L}^{2}\left(\omega_{T}\right)}^{2} \leq C\left\|\mathbf{v}_{\epsilon_{m}}\right\|_{\mathbf{L}^{2}\left(\omega_{T}\right)}^{2} .
$$

Here the assertion follows by the application of (5.7) with $s=\gamma=2$ and (6.8) with $s=2$, and observing that $2<q_{a}^{*}$ by the assumption (3.10). 
- $\lim \sup _{m \rightarrow \infty}\left|J_{7}\right| \leq C 2^{-j}$. Using (7.27), arguing as we did for $\left|J_{4}^{i}\right|$ and using the definition of $E_{m, j}$ (see (7.19)), we can prove that

$$
\begin{aligned}
\limsup _{m \rightarrow \infty}\left|J_{7}\right| & \leq C \limsup _{m \rightarrow \infty}\left[\varrho_{m, j}^{-1} \mathcal{L}_{N+1}\left(E_{m, j}\right)\left(\lambda_{m, j}+\varrho_{m, j}^{-1} \delta_{d, \omega_{T}^{\xi}}^{-N-3}\left\|\mathbf{v}_{\epsilon_{m}}\right\|_{\mathbf{L}^{1}\left(E_{m, j}\right)}\right)^{2}\right] \\
& \leq C \limsup _{m \rightarrow \infty}\left[\varrho_{m, j}^{-1} \mathcal{L}_{N+1}\left(E_{m, j}\right) \lambda_{m, j}^{2}\right] \leq C \limsup _{m \rightarrow \infty}\left[\varrho_{m, j}^{-1} \mathcal{L}_{N+1}\left(E_{m, j}^{i}\right) \lambda_{m, j}^{2}\right] .
\end{aligned}
$$

Then, by the definition of $\varrho_{m, j}$ and (7.15) together with (7.2) and (7.3), we get $\limsup _{m \rightarrow \infty}\left|J_{7}\right| \leq C 2^{-j}$. As a consequence, claim (8.2) follows.

On the other hand, arguing as we did for $\left|J_{4}^{i}\right|$, we can prove also that, for a fixed $j$,

$$
\limsup _{m \rightarrow \infty} \sum_{i=1}^{N}\left|\int_{E_{m, j}}\left(\left|D_{i} \mathbf{u}_{\epsilon_{m}}\right|^{q_{i}-2} D_{i} \mathbf{u}_{\epsilon_{m}}-\mathbf{S}_{i}\right) \otimes \mathbf{e}_{i}: \nabla\left(\mathcal{T}_{m, j}\left(\mathbf{v}_{\epsilon_{m}}\right)\right) \xi d \mathbf{x} d t\right| \leq C 2^{-\frac{j}{\beta}} .
$$

In consequence, from the definition of $\boldsymbol{T}_{m, j}$ (see (7.20)), (8.2) and (8.3), we prove that

$$
\limsup _{m \rightarrow \infty} \sum_{i=1}^{N}\left|\int_{\omega_{T} \backslash E_{m, j}}\left(\left|D_{i} \mathbf{u}_{\epsilon_{m}}\right|^{q_{i}-2} D_{i} \mathbf{u}_{\epsilon_{m}}-\mathbf{S}_{i}\right) \otimes \mathbf{e}_{i}: \nabla\left(\mathcal{T}_{m, j}\left(\mathbf{v}_{\epsilon_{m}}\right)\right) \xi d \mathbf{x} d t\right| \leq C 2^{-\frac{j}{\beta}}
$$

Using the definition of $\mathbf{v}_{\epsilon_{m}}$ (see (6.9)) and the strong convergence property of $p_{\epsilon_{m}}^{h}$ (see (6.8)), it can be derived from (8.4) that

$$
\limsup _{m \rightarrow \infty} \sum_{i=1}^{N}\left|\int_{\omega_{T} \backslash E_{m, j}}\left(\left|D_{i} \mathbf{u}_{\epsilon_{m}}\right|^{q_{i}-2} D_{i} \mathbf{u}_{\epsilon_{m}}-\mathbf{S}_{i}\right) \otimes \mathbf{e}_{i}: \nabla\left(\mathbf{u}_{\epsilon_{m}}-\mathbf{u}\right) \xi d \mathbf{x} d t\right| \leq C 2^{-\frac{j}{\beta}}
$$

In the final part of the proof, we consider an arbitrary $i \in\{1, \ldots, N\}$. Due to (8.5) and (7.11), and since $E_{m, j} \subset E_{m, j}^{i}$, for each $j \in \mathbb{N}$ we can find a number $m_{j} \in \mathbb{N}$ such that

$$
\begin{gathered}
\left|\int_{\omega_{T} \backslash E_{m_{j}, j}^{i}}\left(\left|D_{i} \mathbf{u}_{\epsilon_{m}}\right|^{q_{i}-2} D_{i} \mathbf{u}_{\epsilon_{m}}-\mathbf{S}_{i}\right) \otimes \mathbf{e}_{i}: \nabla\left(\mathbf{u}_{\epsilon_{m_{j}}}-\mathbf{u}\right) \xi d \mathbf{x} d t\right| \leq C 2^{-\frac{j}{\beta}}, \\
\mathcal{L}_{N+1}\left(F_{m_{j}, j}\right) \leq C 2^{-j} \quad \text { and } \quad \mathcal{L}_{N+1}\left(H_{m_{j}, j}^{i}\right) \leq C 2^{-j} .
\end{gathered}
$$

Setting $\xi_{j}^{i}:=\xi \chi_{\omega_{T} \backslash E_{m_{j}, j}^{i}}$, where $\chi_{\omega_{T} \backslash E_{m_{j}, j}^{i}}$ denotes the characteristic function of the set $\omega_{T} \backslash E_{m_{j}, j}^{i}$, it can be proved (see [7, pp. 36-37]), using (7.7), (8.7) and the fact that $\lambda_{m_{j}, j} \geq 1$, that

$$
\xi_{j}^{i} \longrightarrow \xi \quad \text { a.e. in } \omega_{T} \text { as } j \rightarrow \infty .
$$

From (8.8), (4.14) and (4.15), we have, by appealing to Lebesgue's theorem of dominated convergence, that

$$
\begin{aligned}
& D_{i} \mathbf{u} \xi_{j}^{i} \longrightarrow D_{i} \mathbf{u} \xi \quad \text { in } \quad \mathbf{L}^{q_{i}}\left(\omega_{T}\right), \quad \text { as } j \rightarrow \infty \\
& \mathbf{S}_{i} \xi_{j}^{i} \longrightarrow \mathbf{S}_{i} \xi \quad \text { in } \quad \mathbf{L}^{q_{i}^{\prime}}\left(\omega_{T}\right), \quad \text { as } j \rightarrow \infty
\end{aligned}
$$

Then, from (8.6)-(8.7) and (8.9)-(8.10), and appealing once more to (4.14), (4.15) and Lebesgue's theorem of dominated convergence, we obtain

$$
\lim _{j \rightarrow \infty} \int_{\omega_{T}}\left|D_{i} \mathbf{u}_{\epsilon_{m}}\right|^{q_{i}-2} D_{i} \mathbf{u}_{\epsilon_{m}} \otimes \mathbf{e}_{i}: \nabla \mathbf{u}_{\epsilon_{m_{j}}} \xi_{j}^{i} d \mathbf{x} d t=\int_{\omega_{T}} \mathbf{S}_{i} \otimes \mathbf{e}_{i}: \nabla \mathbf{u} \xi d \mathbf{x} d t
$$

for all $i \in\{1, \ldots, N\}$. Finally, taking into account (4.14), (4.15), (8.8) and (8.11), we can use the Minty trick in the spirit of $\left[28\right.$, Lemma A.2] to establish that $\mathbf{S}_{i} \xi=\left|D_{i} \mathbf{u}\right|^{q_{i}-2} D_{i} \mathbf{u} \xi$ a.e. in $\omega_{T}$. Due to the arbitrariness of $\xi, \mathbf{S}_{i}=\left|D_{i} \mathbf{u}\right|^{q_{i}-2} D_{i} \mathbf{u}$ a.e. in $\omega_{T}$, which concludes the proof of Theorem 3.1.

Remark 8.1. It is possible to consider unbounded domains $\Omega$. In this case, proceeding as in Wolf [28, Section 3], we can prove that the regularized problem (4.1)-(4.4) has a unique weak solution for such $\Omega$. As a consequence the original problem has a solution for these domains as well. 


\section{Analysis of the existence Result}

We recall that the main restrictions on the lower bounds of the anisotropic exponents $q_{i}$ to prove Theorem 3.1 are stated at (3.7) and (3.10),

$$
q_{a}^{*}>\beta \quad \text { and } \quad q_{a}^{*}>2 .
$$

As mentioned in Section 3, due to (2.4) and to Remark 2.1, we have

$$
q_{a}^{*}>\beta \Rightarrow \bar{q}^{*}>\beta \Leftrightarrow \bar{q}>\frac{N \beta}{N+\beta} \Leftrightarrow \sum_{j=1}^{N} \frac{1}{q_{j}}<1+\frac{N}{\beta} .
$$

In this case, the second relation of (9.1) reads as

$$
q_{a}^{*}>2 \Leftrightarrow \bar{q}^{*}>2 \Leftrightarrow \bar{q}>\frac{2 N}{N+2} \Leftrightarrow \sum_{j=1}^{N} \frac{1}{q_{j}}<1+\frac{N}{2} .
$$

Combining (9.2) with (9.3), lead us to

$$
\sum_{j=1}^{N} \frac{1}{q_{j}}<\min \left\{1+\frac{N}{\beta}, 1+\frac{N}{2}\right\}=\left\{\begin{array}{lll}
1+\frac{N}{\beta}, & \text { if } & \beta>2, \\
1+\frac{N}{2}, & \text { if } \quad \beta \leq 2 .
\end{array}\right.
$$

Let us consider the case of $\beta \leq 2$ which corresponds to the most difficult situation to analyze. Note that in the isotropic problem, i.e. when $q_{i}=q$ for all $i \in\{1, \ldots, N\}$, the range $1<q<2$ corresponds to the shear-thinning case for which the result of [7] has still left a gap, in the case of $1<q \leq \frac{2 N}{N+2}$ and $N \geq 3$. The following example is very clear about the scope of the outcome established in Theorem 3.1.

Example 9.1. Let us consider $\beta \leq 2$ and $q_{1}=q_{2}=\cdots=q_{N-1}=\alpha$ but distinct of $q_{N}=\beta$. In this case, (3.7) and (3.10) are satisfied if

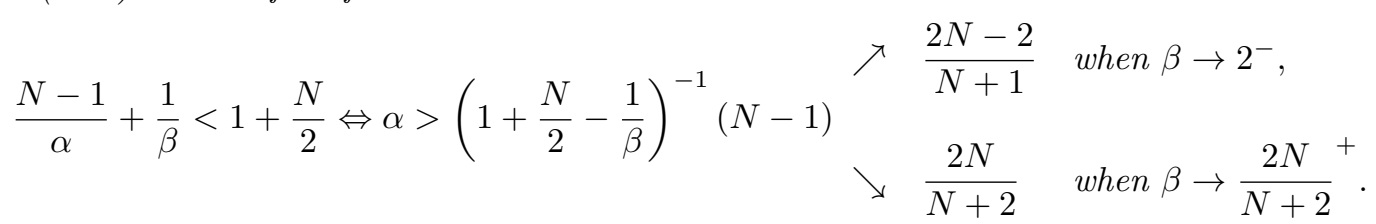

Observe that when $\beta \rightarrow \frac{2 N}{N+2}^{+}$, we end up with $\alpha>\frac{2 N}{N+2}$ which in a certain sense corresponds to assume that $q_{i}=q$ for all $i \in\{1, \ldots, N\}$ and to the recovery of the lower bound $q>\frac{2 N}{N+2}$ for the isotropic problem analyzed in [7]. But, when $\beta \rightarrow 2^{-}$in the particular case of $N=3$, we achieve the optimal condition $\alpha>1$, which means that if for one component, say $q_{3}$, we have $q_{3}=2$, then the other two components satisfy the optimal condition $q_{i}>1$, for $i=1,2$, and as long as $q_{2}=q_{1}$.

The example above shows that Theorem 3.1 improves the result of [7] in order to be optimal in some directions of anisotropy, provided that in other directions there is a compensation. Theorem 3.1 improves also the result [3, Theorem 3.1] for the same anisotropic problem.

\section{REFERENCES}

[1] S.N. Antontsev and H.B. de Oliveira. Finite time localized solutions of fluid problems with anisotropic dissipation. Internat. Ser. Numer. Math. 154, Birkhäuser (2006), pp. 23-32.

[2] S.N. Antontsev and H.B. de Oliveira. Analysis of the existence for the steady Navier-Stokes equations with anisotropic diffusion. Adv. Differential Equations 19 (2014) no. 5-6, 441-472.

[3] S.N. Antontsev and H.B. de Oliveira. Evolution problems of Navier-Stokes type with anisotropic diffusion. RACSAM - Revista de la Real Academia de Ciencias Exactas, Físicas y Naturales. Serie A. Matemáticas 110 (2016), no. 2, 729-754.

[4] J.-Y. Chemin, B. Desjardins, I. Gallagher and E. Grenier. Fluids with anisotropic viscosity. Special issue for R. Temam's 60th birthday. M2AN Math. Model. Numer. Anal. 34 (2000), no. 2, 315-335. 
[5] J.-Y. Chemin and P. Zhang. On the global wellposedness to the 3-D incompressible anisotropic NavierStokes equations. Comm. Math. Phys. 272 (2007), no. 2, 529-566.

[6] B. Cushman-Roisin and J.-M. Beckers. Introduction to geophysical fluid dynamics. Physical and numerical aspects. Second ed. Elsevier/Academic Press, Amsterdam, 2011.

[7] L. Diening, M. Rǔžička and J. Wolf. Existence of weak solutions for unsteady motions of generalized Newtonian fluids. Ann. Scuola Norm. Sup. Pisa Cl. Csi. 5, IX (2010), 1-46.

[8] I. Fragalà, F. Gazzola and B. Kawohl. Existence and nonexistence results for anisotropic quasilinear elliptic equations. Ann. Inst. H. Poincaré Anal. Non Linéaire 21 (2004), no. 5, 715-734.

[9] G.P. Galdi. An introduction to the mathematical theory of the Navier-Stokes equations. Steady-state problems. Springer, New York, 2011.

[10] J. Haškovec and C. Schmeiser. A note on the anisotropic generalizations of the Sobolev and Morrey embedding theorems. Monatsh. Math. 158 (2009), no. 1, 71-79.

[11] D. Iftimie. The resolution of the Navier-Stokes equations in anisotropic spaces. Rev. Mat. Iberoamericana 15 (1999), no. 1, 1-36.

[12] D. Iftimie. A uniqueness result for the Navier-Stokes equations with vanishing vertical viscosity. SIAM J. Math. Anal. 33 (2002) 1483-1493.

[13] O.A. Ladyzhenskaya. New equations for the description of motion of viscous incompressible fluids and solvability in the large of boundary value problem for them. Proc. Steklov Inst. Math 102 (1967), 95-118.

[14] O.A. Ladyzhenskaya. The mathematical theory of viscous incompressible flow. Gordon and Breach, Science Publishers, New York-London-Paris, 1969.

[15] J.-L. Lions. Quelques mèthodes de résolution des problèmes aux limites non liniaires. Dunod, Paris, 1969.

[16] J. Málek, J. Nečas, M. Rokyta and M.Rǔžička. Weak and measure-valued solutions to evolutionary PDEs. Applied Mathematics and Mathematical Computation, 13. Chapman \& Hall, London, 1996.

[17] W. Ostwald. Ueber die Geschwindigkeitsfunktion der Viskositat Disperser Systeme, I. Kolloid-Z. 36 (1925), 99-117.

[18] M. Paicu. Équation anisotrope de Navier-Stokes dans des espaces critiques. Rev. Mat. Iberoam. 21 (2005) 179-235.

[19] M. Paicu and P. Zhang. Global solutions to the 3-D incompressible anisotropic Navier-Stokes system in the critical spaces. Comm. Math. Phys. 307 (2011) 713-759.

[20] J. Pedlosky. Geophysical fluid dynamics. Second ed. Springer-Verlag, New-York, 1987.

[21] P. Penel and M. Pokorný. Improvement of some anisotropic regularity criteria for the Navier-Stokes equations. Discrete Contin. Dyn. Syst. Ser. S 6 (2013), no. 5, 1401-1407.

[22] M.M. Porzio. $\mathrm{L}^{\infty}$-regularity for degenerate and singular anisotropic parabolic equations. Boll. Un. Mat. Ital. A 11, no. 7, 697-707 (1997).

[23] J. Rákosník. Some remarks to anisotropic Sobolev spaces. II. Beiträge Anal. No. 15 (1980), 127-140 (1981).

[24] K.N. Soltanov. On some modification Navier-Stokes equations. Nonlinear Anal., Theory Methods Appl., Ser. A 52 (2003), no. 3, 769-793.

[25] E.M. Stein. Singular integrals and diferentiability properties of functions. Princeton University Press, 1970.

[26] M. Troisi. Teoremi di inclusione per spazi di Sobolev non isotropi. Ricerche Mat. 18 (1969), 3-24.

[27] A. de Waele. Viscometry and plastometry. J. Oil Color Chem. Assoc. 6 (1923), 33-88.

[28] J. Wolf. Existence of weak solutions to the equations of non-stationary motion of non-Newtonian fluids with shear rate dependent viscosity. J. Math. Fluid Mech. 9 (2007), no. 1, 104-138.

[29] K. Yan and Z. Yin. Global well-posedness of the three dimensional incompressible anisotropic NavierStokes system. Nonlinear Anal. Real World Appl. 32 (2016), 52-73.

[30] T. Zhang and D. Fang. Global wellposed problem for the 3-D incompressible anisotropic Navier-Stokes equations. J. Math. Pures Appl. 90 (2008) 413-449.

[31] V. Zhikov. New approach to the solvability of generalized Navier-Stokes equations. Funct. Anal. Appl. 43 (2009), no. 3, 190-207. 